\begin{tabular}{|c|l|}
\hline Title & On a denominator of a certain formal power series attached to local densities \\
\hline Author(s) & Hisasue, Masaki \\
\hline Citation & 北海道大学. 博士(理学) 甲第3987号 \\
\hline Issue Date & 1997-03-25 \\
\hline DOI & 10.11501/3122145 \\
\hline Doc URL & http://hdl.handle.net/2115/51400 \\
\hline Type & theses (doctoral) \\
\hline File Information & 000000307336.pdf \\
\hline
\end{tabular}

Instructions for use 
On a denominator of a certain formal power series attached to local densities 


\section{On a denominator of a certain formal power series attached to local densities}

\section{MASAKI Hisasue}

Department of Mathematics, Hokkaido University, Sapporo 060 Japan

\section{Introduction}

Let $p$ be a prime number. When $A$ and $B$ are non-degenerate symmetric matrices over $\mathbb{Z}_{p}$ of degree $m$ and $n(m \geq n)$, respectively, we define the local density $\alpha_{p}(B, A)$ by

$$
\alpha_{p}(B, A)=\lim _{e \rightarrow \infty} p^{-t e} \# A_{p^{e}}(B, A)
$$

where $t=m n-n(n+1) / 2, A_{p^{e}}(B, A)=\left\{\bar{X} \in M_{m n}\left(\mathbb{Z}_{p}\right) / p^{e} M_{m n}\left(\mathbb{Z}_{p}\right) ; A[X] \equiv B\right.$ $\left.\bmod p^{e}\right\}$, and $A[X]={ }^{t} X A X$.

In spite of the importance of local densities, it is not easy to calculate their explicit values.

In [9], Kitaoka introduced the formal power series

$$
P(B, A ; x)=\sum_{r=0}^{\infty} \alpha_{p}\left(p^{r} B, A\right) x^{r}
$$

He proved it is a rational function of $x$ and obtained its denominator for $A=$ $\left(\begin{array}{cc}O_{k} & E_{k} \\ E_{k} & O_{k}\end{array}\right)$ where $E_{k}$ (resp. $O_{k}$ ) is the identity matrix of degree $k$ (resp. the zero matrix of degree $k$ ). Moreover he conjectured the rationality for an arbitrary symmetric $A$. Its rationality and the denominator have been investigated by Böcherer-Sato, Hironaka, and Katsurada in special cases.

In [2], Hironaka proved the rationality and the determined its denominator for arbitrary $A$ and $B$ in the case $p \neq 2$.

Böcherer and Sato ([1]) showed the rationality of the series for arbitrary $A$ and $B$. They also determined its denominator in the following two cases by applying Denef's theory of $p$-adic integrals;

(1) $m$ is even and $A$ is unimodular,

(2) $B$ is anisotropic. 
When $B$ is decomposed into the form $B=B_{0} \perp \cdots \perp B_{s}$, they generalized (0.2) and defined a formal power series of several variables

$$
\begin{aligned}
& P\left(\left(B_{0}, B_{1}, \ldots, B_{s}\right), A ; x_{1}, \ldots, x_{s}\right) \\
& \quad=\sum_{r_{1}, \ldots, r_{s}=0}^{\infty} \alpha_{p}\left(B_{0} \perp p^{r_{1}} B_{1} \perp \cdots \perp p^{r_{s}} B_{s}, A\right) x_{1}^{r_{1}} \ldots x_{s}^{r_{s}} .
\end{aligned}
$$

If $s=1$ and $\operatorname{deg}\left(B_{0}\right)=0$, then this is nothing but the previous one. They proved that this represents a rational function of $x_{1}, \ldots, x_{l}$ and that every factor of the denominator is of the form

$$
1-p^{-c} x_{1}^{a_{1}} \ldots x_{s}^{a_{s}} \quad\left(c, a_{1}, \ldots, a_{s} \in \mathbb{Z}\right) .
$$

In [3], Katsurada introduced the local density with a congruence condition and proved the rationality of $(0.3)$ in the case that $\operatorname{deg}\left(B_{0}\right)=0$. He also obtained the explicit denominator for any diagonal matrix $B$ in the case $p \neq 2$ and $\operatorname{deg}\left(B_{0}\right)=0$. Moreover, in [4], he modified formal power series into

$$
\begin{aligned}
& R\left(\left(B_{1}, \ldots, B_{s}\right), A ; x_{1}, \ldots, x_{s}\right) \\
& \quad=\sum_{r_{1} \geq \cdots \geq r_{s} \geq 0} \alpha_{p}\left(p^{r_{1}} B_{1} \perp \cdots \perp p^{r_{s}} B_{s}, A\right) x_{1}^{r_{1}} \ldots x_{s}^{r_{s}},
\end{aligned}
$$

where $B=B_{1} \perp \cdots \perp B_{s}, \operatorname{deg}\left(B_{i}\right)=n_{i}$. He and the present author proved the rationality of the series when $A$ is even unimodular and $B$ is diagonal in [5].

In the present paper, we show the rationality and determine the denominators of this series when $A$ and $B$ are arbitrary matrices. We would like to emphasize the method for $p \neq 2$ in [3] cannot be directly applied to the odd unimodular case.

We define the following formal power series;

$$
\begin{aligned}
& Q\left(\left(B_{0}, B_{1}, \ldots, B_{s}\right), A ; x_{1}, \ldots, x_{s}\right) \\
& \quad=\sum_{r_{1} \geq \cdots \geq r_{s} \geq 0} \alpha_{p}\left(B_{0} \perp p^{r_{1}} B_{1} \perp \cdots \perp p^{r_{s}} B_{s}, A\right) x_{1}^{r_{1}} \ldots x_{s}^{r_{s}} .
\end{aligned}
$$

Note that

$$
Q\left(\left(\varnothing, B_{1}, \ldots, B_{s}\right), A ; x_{1}, \ldots, x_{s}\right)=R\left(\left(B_{1}, \ldots, B_{s}\right), A ; x_{1}, \ldots, x_{s}\right) .
$$

Our main results are as follows:

Theorem 1. Let $A$ and $B$ be are arbitrary non-degenerate symmetric matrices as above. Let $t$ be the Witt index of $A$. Put

$$
u_{k}=\min \left(n_{1}+\cdots+n_{k}-1, t\right)
$$

and

$$
v_{k}= \begin{cases}1 & t \geq n_{1}+\cdots+n_{k} \\ 0 & \text { otherwise }\end{cases}
$$


Then $P\left(\left(B_{0}, B_{1}, \ldots, B_{s}\right), A ; x_{1}, \cdots, x_{s}\right)$ is a rational function of $x_{1}, \ldots, x_{s}$ with a denominator

$$
\prod_{k=1}^{s}\left(1-x_{1} \ldots x_{k}\right)^{v_{k}} \prod_{i=0}^{u_{k}}\left(1-p^{\left(n_{1}+\cdots+n_{k}-i\right)(-m+n+i+1)}\left(x_{1} \ldots x_{k}\right)^{2}\right) .
$$

Theorem 1, in the case $A$ is even unimodular and $\operatorname{deg}\left(B_{0}\right)=0$, has been proved by Katsurada and the present author in [6]. The crucial part of the present paper is to prove Theorem 1 when $A$ is odd unimodular.

Remark that the value of the local density coincides with the special value of singular series, hence it is meaningful to study not only the even unimodular case but also the arbitrary case.

If $s=1$ and $B_{0}=\varnothing$, then by definition $P(B, A ; x)=Q((\varnothing, B), A ; x)$.

Corollary. The series $P(B, A ; x)$ is a rational function of $x$ with a denominator

$$
(1-x)^{v} \prod_{i=0}^{u}\left(1-p^{(n-i)(-m+n+i+1)} x^{2}\right)
$$

where $u=\min (n-1, t)$ and $v=1$ or 0 according as $t \geq n$ or not.

Since $P\left(\left(B_{0}, B_{1}, \ldots, B_{s}\right), A ; x_{1}, \cdots, x_{s}\right)$ is a linear combination of the series similar to $Q\left(\left(B_{0}, B_{1}, \ldots, B_{s}\right), A ; x_{1}, \cdots, x_{s}\right)$, we have the following:

Theorem 2. Let $A, B, t, u_{k}$ and $v_{k}$ be as above. Then $P\left(\left(B_{0}, B_{1}, \ldots, B_{s}\right), A ; x_{1}, \cdots, x_{s}\right)$ is a rational function of $x_{1}, \ldots, x_{s}$ with a denominator

$$
\prod_{k=1}^{s}\left(1-x_{1} \ldots x_{k}\right)^{v_{k}} \prod_{i=0}^{u_{k}}\left(1-p^{\left(n_{1}+\cdots+n_{k}-i\right)(-m+n+i+1)}\left(x_{1} \ldots x_{k}\right)^{2}\right)
$$

Notation. For a set $Z, \# Z$ denotes the cardinality of $Z$. For a commutative ring with an identity element $R$, we denote by $M_{m n}(R)$ the ring of $(m, n)$-matrices with entries in $R$. Put $M_{m}(R)=M_{m m}(R)$. When $m=0$ or $n=0$, we understand $M_{m n}(R)=\varnothing . G L_{m}(R)$ is the group of all invertible elements of $M_{m}(R)$. We call elements of $G L_{m}(R)$ unimodular. Further let $S_{n}(R)$ denote the set of all symmetric matrices in $M_{n}(R)$. Put $\mathbb{Z}_{\geq 0}=\{x \in \mathbb{Z} ; x \geq 0\}$. By $\mathbb{Q}_{p}$ and $\mathbb{Z}_{p}$ we denote the $p$-adic number field and the ring of $p$-adic integers, respectively. For any square matrix $A$, $\operatorname{tr}(A)$ denotes the trace of $A$. For a symmetric unimodular matrix $A$ with entries in $\mathbb{Z}_{p}$, we say $A$ is even unimodular if all the diagonal elements belong to $2 \mathbb{Z}_{p}$, otherwise we say $A$ is odd unimodular.

Let $M$ be a $R$-module and $Q$ a mapping from $M$ to $R$ which satisfies the conditions (1) $Q(r m)=r^{2} Q(m)$ for $r \in R, m \in M,(2) 2 B(m, n):=Q(m+n)-Q(m)-$ $Q(n)$ is a symmetric bilinear form. We call $(M, Q)$ or simply $M$ a quadratic module over $R, Q$ a quadratic form and $B$ an associated symmetric bilinear form. For quadratic modules $M$ and $N$ over $\mathbb{Z}_{p}$, if they are isometric, we write $M \simeq N$. For 
square matrices $U$ and $V$, we denote $\left(\begin{array}{cc}U & O \\ O & V\end{array}\right)$ by $U \perp V$. For an element $a$ of $R$ and an element $X$ of $M_{m n}(R)$, we often use the same symbol $X$ for the class $X$ modulo $a M_{m n}(R)$. We denote by $1_{m n}$ (resp. $\left.0_{m n}\right)$ the $(m, n)$-matrices whose components are all 1 (resp. 0). For any matrices $A, B \in M_{n}\left(\mathbb{Z}_{p}\right)$, we say $A$ and $B$ are $\mathbb{Z}_{p}$-equivalent if there exists a matrix $X \in G L_{n}\left(\mathbb{Z}_{p}\right)$ satisfying $A[X]=B$. Finally, for an element $a$ of $\mathbb{Q}_{p}, \nu$ denotes the integer $n$ such that $a \in p^{n} \mathbb{Z}_{p}$ and $a \notin p^{n+1} \mathbb{Z}_{p}$.

\section{Preliminary results}

For $S \in S_{m}\left(\mathbb{Z}_{p}\right), T \in S_{n}\left(\mathbb{Z}_{p}\right), \Xi=\left(\xi_{i}\right) \in\left(\mathbb{Z}_{p} / p^{e} \mathbb{Z}_{p}\right)^{m}$, and a non-negative integer $e$, we put (cf. [2])

$$
A_{e}(T, S ; \Xi)=\left\{X=\left(x_{i j}\right) \in A_{e}(T, S) ; x_{i 1} \equiv \xi_{i} \bmod p^{e} \text { for } 1 \leq i \leq m\right\}
$$

and

$$
a_{e}(T, S ; \Xi)=\# A_{e}(T, S ; \Xi) .
$$

For a non-negative integer $r<m+n$, put

$$
A_{e}(r ; T, S)=\left\{\bar{X} \in M_{m+n-r}\left(\mathbb{Z}_{p} / p^{e} \mathbb{Z}_{p}\right) ;(S \perp-T)\left[\left(\begin{array}{c}
X \\
E_{r}
\end{array}\right)\right] \equiv O_{r} \quad \bmod p^{e}\right\}
$$

where $E_{r}$ is the identity matrix of degree $r$. Put

$$
a_{e}(r ; T, S)=\# A_{e}(r ; T, S)
$$

Moreover, put

$$
\alpha_{p}(r ; T, S)=\lim _{e \rightarrow \infty} p^{-e(((m+n-r) r-<r>)} a_{e}(r ; T, S) .
$$

This limit exists as will be shown in Theorem 3.2. Note that if $T=t \perp T_{1}$, then we have $\alpha_{p}(n-1 ; T, S)=\alpha_{p}\left(T_{1}, S \perp(-t)\right)$.

Put $H=\left(\begin{array}{ll}0 & 1 \\ 1 & 0\end{array}\right)$ and $Y=\left(\begin{array}{ll}2 & 1 \\ 1 & 2\end{array}\right)$. If $p \neq 2$, it is well known that a nondegenerate symmetric matrix is equivalent over $\mathbb{Z}_{p}$ to a unique matrix of the following form:

$$
\perp_{i=0}^{r} p^{i}\left(V_{i} \perp U_{i}\right)
$$

where

$$
V_{i}=\overbrace{H \perp \cdots \perp H}^{l_{i}} \text { with } l_{i} \geq 0
$$

and

$$
U_{i}=\varnothing,(c) \text { or } c_{1} \perp c_{2} \text { with } c, c_{1}, c_{2} \in \mathbb{Z}_{p}^{\times} \text {and }-c_{1} c_{2} \notin\left(\mathbb{Z}_{p}^{\times}\right)^{2} .
$$

Here $\varnothing$ denotes the empty matrix. 
If $p=2$, we review the result of Watson [12] in a modified way (cf.[6]). A nondegenerate symmetric matrix with entries in $\mathbb{Z}_{2}$ is equivalent over $\mathbb{Z}_{2}$ to a unique matrix of the following form:

$$
\perp_{i=0}^{r} 2^{i}\left(V_{i} \perp U_{i}\right)
$$

where

$$
V_{i}=\overbrace{H \perp \cdots \perp H}^{l_{i}} \perp Y_{i} \text { with } l_{i} \geq 0 \text { and } Y_{i}=\varnothing \text { or } Y
$$

and

$$
U_{i}=\perp_{j=1}^{k_{i}} c_{i j} \text { with } c_{i j} \in \mathbb{Z}_{2}^{\times}, 0 \leq k_{i} \leq 2
$$

satisfying the following conditions:

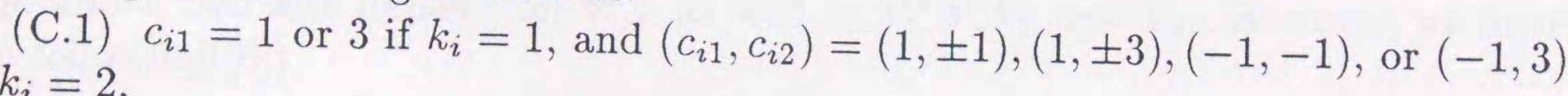
if $k_{i}=2$,

(C.2) $k_{i}=k_{i-2}=0$ if $Y_{i-1}=Y$,

(C.3) $-\operatorname{det} U_{i} \equiv 1 \bmod 4$ if $Y_{i}=Y$ and $k_{i}=2$,

(C.4) $(-1)^{k_{i}-1} \operatorname{det} U_{i} \equiv 1 \bmod 4$ if $k_{i+1} \neq 0, k_{i} \neq 0$,

(C.5) $U_{i} \neq-1 \perp-c_{i 2}$ if $k_{i-1} \neq 0$,

(C.6) $U_{i}=\varnothing,(1), 1 \perp \pm 1,-1 \perp-1$ if $k_{i+2} \neq 0$.

The matrix satisfying the above conditions (C.1) (C.6) is said to be canonical form. Let the notation be as above, and $i_{1}, \ldots, i_{s}$ be non-negative integers such that $i_{1}<\cdots<i_{s} \leq r$ and $\operatorname{deg}\left(U_{i_{k}} \perp V_{i_{k}}\right) \geq 1$ for $k=1, \ldots, s$. Then we call $\left(i_{1}, \ldots, i_{s}\right)$ the exponent.

For $\mathbb{Z}_{p}$-modules $V$ and $W$, let $\operatorname{Hom}_{\mathbb{Z}_{p}}(W, V)$ be the set of $\mathbb{Z}_{p}$-homomorphisms from $W$ to $V$. For an element $\phi$ of $\operatorname{Hom}_{\mathbb{Z}_{p}}(W, V)$, we use the same symbol $\phi$ for the image of the natural projection $\pi: \operatorname{Hom}_{\mathbb{Z}_{p}}(W, V) \longrightarrow \operatorname{Hom}_{\mathbb{Z}_{p}}(W, V) \otimes \mathbb{Z}_{p} / p^{e} \mathbb{Z}_{p}$. For quadratic modules $V, W$ over $\mathbb{Z}_{p}$ and an integer $e \geq 0$, we set

$L_{e}(W, V)$

$=\left\{\phi \in \operatorname{Hom}_{\mathbb{Z}_{p}}(W, V) \otimes \mathbb{Z}_{p} / p^{e} \mathbb{Z}_{p} ; B\left(\phi(w), \phi\left(w^{\prime}\right)\right) \equiv B\left(w, w^{\prime}\right) \bmod p^{e}\right.$ for $\left.w, w^{\prime} \in W\right\}$.

Further, for a quadratic submodule $W_{1}$ of $W$ and $\phi_{1} \in L_{e}\left(W_{1}, V\right)$ put

$$
L_{e}\left(W, V ; \phi_{1}\right)=\left\{\phi \in L_{e}(W, V) ; \phi \mid W_{1} \equiv \phi_{1} \bmod p^{e} V\right\},
$$

where $\phi \mid W_{1}$ denotes the restriction of $\phi$ to $W_{1}$.

Let $V=\mathbb{Z}_{p}\left[v_{1}, \ldots, v_{m}\right]$ and $W=\mathbb{Z}_{p}\left[w_{1}, \ldots, w_{n}\right]$ be quadratic modules over $\mathbb{Z}_{p}$ satisfying $\left(B\left(v_{i}, v_{j}\right)\right)=S,\left(B\left(w_{i}, w_{j}\right)\right)=T$. Put $\phi_{1}\left(w_{1}\right)=\sum_{i=1}^{m} \xi_{i} v_{i}$ with $\xi_{i} \in \mathbb{Z}_{p}$.
Then it is easy to see that

$$
\# L_{e}(W, V)=\# A_{e}(T, S)
$$

and

$$
\# L_{e}\left(W, V ; \phi_{1}\right)=\# A_{e}\left(T, S ;\left(\xi_{i}\right)\right)
$$


Let $V$ and $W$ be as above, and $V_{1}, V_{2}$ be quadratic submodules of $V$ such that $V=V_{1} \oplus V_{2}$. Assume that the $\mathbb{Z}_{p}$-rank of $V_{2}$ and $W$ is equal. Let $\mathbf{v}=\left\{v_{1}, \ldots, v_{n}\right\}$, $\mathbf{w}=\left\{w_{1}, \ldots, w_{n}\right\}$ be the $\mathbb{Z}_{p}$-basis of $V_{2}$ and $W$, respectively. Then we set

$$
L_{e}\left(W, V, V_{1}, V_{2} ; \mathbf{w}, \mathbf{v}\right)=\left\{\phi \in L_{e}(W, V) ; \operatorname{Pr}_{V \rightarrow \mathbb{Z}_{p}\left[v_{i}\right]} \phi\left(w_{j}\right)=\delta_{i j} v_{i} \text { for } 1 \leq i, j \leq n\right\},
$$

where $\delta_{i j}$ is the Kronecker's delta and $\operatorname{Pr}_{V \rightarrow \mathbb{Z}_{p}\left[v_{i}\right]}$ denotes the projection of $V$ to $\mathbb{Z}_{p}\left[v_{i}\right]$. Further for $W_{1}=\mathbb{Z}_{p}\left[w_{1}\right]$ and $\phi_{1} \in L_{e}\left(W_{1}, V\right)$ such that $\operatorname{Pr}_{V \rightarrow \mathbb{Z}_{p}\left[v_{i}\right]} \phi_{1}\left(w_{1}\right)=$ $\delta_{i 1} v_{i}(1 \leq i \leq n)$ put

$$
L_{e}\left(W, V ; V_{1}, V_{2} ; \mathbf{w}, \mathbf{v} ; W_{1}, \phi_{1}\right)=\left\{\phi \in L_{e}\left(W, V ; V_{1}, V_{2} ; \mathbf{w}, \mathbf{v}\right) ; \phi \mid W_{1}=\phi_{1}\right\} .
$$

The above two sets depend on $\mathbf{w}, \mathbf{v}$ as well as $W, V, V_{1}$ and $V_{2}$. However we have the following $([6])$.

Proposition 1.1. Assume that $W=\mathbb{Z}_{p}\left[w_{1}, \ldots, w_{n}\right]$ is totally singular and $V=$ $V_{1} \perp V_{2}$ where $V_{2}=\mathbb{Z}_{p}\left[v_{1}, \ldots, v_{n}\right]$. Let $V_{2}^{\prime}=\mathbb{Z}_{p}\left[v_{1}^{\prime}, \ldots, v_{n}^{\prime}\right]$ be a quadratic module such that

$$
B\left(v_{i}^{\prime}, v_{j}^{\prime}\right)=-B\left(v_{i}, v_{j}\right) \quad(1 \leq i, j \leq n) .
$$

Then there exists a bijection from $L_{e}\left(W, V ; V_{1}, V_{2} ; \mathbf{w}, \mathbf{v}\right)$ to $L_{e}\left(V_{2}^{\prime}, V_{1}\right)$. Further this induces a bijection from $L_{e}\left(W, V ; V_{1}, V_{2} ; \mathbf{w}, \mathbf{v} ; \mathbb{Z}_{p}\left[v_{1}^{\prime}\right], \phi_{1}\right)$ to $L_{e}\left(V_{2}^{\prime}, V_{1} ; \phi_{1}^{\prime}\right)$ where $\phi_{1} \in L_{e}\left(\mathbb{Z}_{p}\left[w_{1}\right], V\right)$ such that $\operatorname{Pr}_{V \rightarrow \mathbb{Z}_{p}\left[v_{i}\right]} \phi_{1}\left(w_{1}\right)=\delta_{i 1} v_{i}$ for $1 \leq i \leq n$ and $\phi_{1}^{\prime} \in$ $L_{e}\left(\mathbb{Z}_{p}\left[v_{1}^{\prime}\right], V_{1}\right)$ such that $\phi_{1}^{\prime}\left(v_{1}^{\prime}\right)=\operatorname{Pr}_{V \rightarrow V_{1}} \phi_{1}\left(w_{1}\right)$.

\section{Some propositions}

In this section we will prove an essential proposition to get a recursion formula.

Definition. We call an element $T$ of $S_{n}\left(\mathbb{Z}_{p}\right)$ or $\langle T\rangle$ of level $p^{l}$ if $l$ is the least integer such that $p^{l} T^{-1}$ is even integral.

Proposition 2.1([10, p91]). Let $L=\mathbb{Z}_{p}\left[v_{1}, \ldots, v_{n}\right]$ and $M=\mathbb{Z}_{p}\left[w_{1}, \ldots, w_{n}\right]$ be quadratic lattices over $\mathbb{Z}_{p}$ with $\operatorname{rank} L=\operatorname{rank} M=n$ and suppose that $\mathbb{Q}_{p} L$ is regular. Let $h$ be the level of the dual lattice $L^{*}$ of $L$, i.e. $L^{*}=\left\{x \in \mathbb{Q}_{p} L ; B(x, L) \subset \mathbb{Z}_{p}\right\}$. If $B\left(v_{i}, v_{j}\right) \equiv B\left(w_{i}, w_{j}\right) \bmod p^{h+1} \mathbb{Z}_{p}$ and $Q\left(v_{i}\right) \equiv Q\left(w_{i}\right) \bmod 2 p^{h+1} \mathbb{Z}_{p}$, then there exists an isometry $\eta: L \simeq M$ satisfying $\eta\left(v_{i}\right) \equiv w_{i} \bmod p^{h+1} M^{*}$.

Let $A=V \perp U$ be a symmetric unimodular matrix with canonical form. Let $V=\perp_{l} H \perp Y^{\prime}$, where $Y^{\prime}=Y$ or $\varnothing$ and $U=\perp_{j} c_{j}$ as above. Note that if $p \neq 2$, we have always $Y^{\prime}=\varnothing$. Then for $0 \leq r \leq l$ set

$$
A^{(r)}=\perp_{l-r} H \perp Y^{\prime} \perp U \text { and } A^{*}=V .
$$


Lemma 2.2. Let $k_{1}, k_{2}, k_{3}$ and $k$ be non-negative integers such that $k=\sum_{l=1}^{3} k_{l}$. Let $K=\mathbb{Z}_{p}\left[z_{1}, \ldots, z_{k}\right]$ be a quadratic module over $\mathbb{Z}_{p}$ such that

$$
\left(B\left(z_{i}, z_{j}\right)\right)_{1 \leq i, j \leq k}=S \perp T,
$$

with $S=V \perp U$ be a unimodular matrix as above such that $\operatorname{deg} V=k_{1}$ and $\operatorname{deg}$ $U=k_{2}$ and $T=p^{s} T_{1} \perp T_{2} \in M_{k_{3}}\left(\mathbb{Z}_{p}\right)$, where $\operatorname{deg}\left(T_{1}\right) \leq 2$ and $s \geq 2+\delta_{2 p}$. Put $K_{1}=\mathbb{Z}_{p}\left[z_{1}, \ldots, z_{k_{1}}\right], K_{2}=\mathbb{Z}_{p}\left[z_{k_{1}+1}, \ldots, z_{k_{1}+k_{2}}\right]$ and $K_{3}=\mathbb{Z}_{p}\left[z_{k_{1}+k_{2}+1}, \ldots, z_{k}\right]$.

For any elements $u_{l} \in K_{l} \quad(1 \leq l \leq 2)$ such that $u_{1}+u_{2}$ is primitive, we put $z_{1}^{\prime}=u_{1}+u_{2}+z_{k_{1}+k_{2}+1}$.

(1) Assume that $u_{1}$ is primitive and $Q\left(u_{1}+u_{2}\right) \equiv 0 \bmod 2 p$, then there exist elements $\left\{z_{i}^{\prime}\right\}_{2 \leq i \leq k}$ of $K$ such that

(a) $z_{2}^{\prime} \in K_{1}$ and $\left(\begin{array}{ll}B\left(z_{1}^{\prime}, z_{1}^{\prime}\right) & B\left(z_{1}^{\prime}, z_{2}^{\prime}\right) \\ B\left(z_{2}^{\prime}, z_{1}^{\prime}\right) & B\left(z_{2}^{\prime}, z_{2}^{\prime}\right)\end{array}\right)=\left(\begin{array}{cc}\eta_{1} & \eta_{2} \\ \eta_{2} & 0\end{array}\right)$ with $\eta_{1} \in 2 \mathbb{Z}_{p}, \eta_{2} \in \mathbb{Z}_{p}^{\times}$,

(b) $\mathbb{Z}_{p}\left[z_{3}^{\prime}, \ldots, z_{k_{1}+k_{2}}^{\prime}\right]$ is isometric to $\left\langle S^{(1)}\right\rangle$,

(c) $\mathbb{Z}_{p}\left[z_{k_{1}+k_{2}+1}^{\prime}, \ldots, z_{k}^{\prime}\right]$ is isometric to $K_{3}$,

(d) $K=\mathbb{Z}_{p}\left[z_{1}^{\prime}, z_{2}^{\prime}\right] \perp \mathbb{Z}_{p}\left[z_{3}^{\prime}, \ldots, z_{k_{1}+k_{2}}^{\prime}\right] \perp \mathbb{Z}_{p}\left[z_{k_{1}+k_{2}+1}^{\prime}, \ldots, z_{k}^{\prime}\right]$,

(e) $z_{j}^{\prime}-z_{j} \in \mathbb{Z}_{p}\left[z_{1}, \ldots, z_{k_{1}+k_{2}+1}\right]$ for $k_{1}+k_{2}+1 \leq j \leq k$.

(2) Let $p=2$. Assume that $u_{1} \equiv 0 \bmod p$ and $Q\left(u_{1}+u_{2}\right) \equiv 0 \bmod p^{3}$, then there exist elements $\left\{z_{i}^{\prime}\right\}_{2 \leq i \leq k}$ of $K$ such that $\mathbb{Z}_{p}^{\times}$

(a) $z_{2}^{\prime} \in K_{2}$ and $\left(\begin{array}{ll}B\left(z_{1}^{\prime}, z_{1}^{\prime}\right) & B\left(z_{1}^{\prime}, z_{2}^{\prime}\right) \\ B\left(z_{2}^{\prime}, z_{1}^{\prime}\right) & B\left(z_{2}^{\prime}, z_{2}^{\prime}\right)\end{array}\right)=\left(\begin{array}{ll}\eta_{1} & \eta_{2} \\ \eta_{2} & \eta_{3}\end{array}\right)$ with $\eta_{1} \in p^{3} \mathbb{Z}_{p}, \eta_{2}, \eta_{3} \in$

(b) $\mathbb{Z}_{p}\left[z_{3}^{\prime}, \ldots, z_{k_{1}+k_{2}}^{\prime}\right]$ is a submodule of $K_{1} \perp K_{2}$ isometric to $\langle V\rangle$,

(c) $\mathbb{Z}_{p}\left[z_{k_{1}+k_{2}+1}^{\prime}, \ldots, z_{k}^{\prime}\right]$ is isometric to $K_{3}$,

(d) $K=\mathbb{Z}_{p}\left[z_{1}^{\prime}, z_{2}^{\prime}\right] \perp \mathbb{Z}_{p}\left[z_{3}^{\prime}, \ldots, z_{k_{1}+k_{2}}^{\prime}\right] \perp \mathbb{Z}_{p}\left[z_{k_{1}+k_{2}+1}^{\prime}, \ldots, z_{k}^{\prime}\right]$,

(e) $z_{j}^{\prime}-z_{j} \in 2 \mathbb{Z}_{p}\left[z_{1}, \ldots, z_{k_{1}+k_{2}+1}\right]$ for $k_{1}+k_{2}+1 \leq j \leq k$.

Proof.

(1) This can be proved by the same argument as that in the proof of $[3$, Lemma 2.3 ] so we omit the proof.

(2) In this case, we may assume that $a_{k_{1}+1} \not \equiv 0 \bmod p$. Then put $z_{2}^{\prime}=z_{k_{1}+1}$. Then such a $z_{2}^{\prime}$ satisfies the statement (a). It is easy to see that

$$
K_{1} \perp K_{2}=\mathbb{Z}_{p}\left[u_{1}+u_{2}, z_{2}^{\prime}, z_{1}, \ldots, z_{k_{1}}\right] .
$$

Then there exist elements $\left\{\mu_{j}\right\}_{1 \leq j \leq k_{1}+k_{2}},\left\{\nu_{j}\right\}_{1 \leq j \leq k_{1}+k_{2}}$ of $\mathbb{Z}_{p}$ such that

$$
B\left(z_{j}+\mu_{j}\left(u_{1}+u_{2}\right)+\nu_{j} z_{2}^{\prime}, z_{i}^{\prime}\right)=0 \quad\left(1 \leq j \leq k_{1}+k_{2}, 1 \leq i \leq 2\right) .
$$

Put $z_{j+2}^{\prime}=z_{j}+\mu_{j}\left(u_{1}+u_{2}\right)+\nu_{j} z_{2}^{\prime}$ for $1 \leq j \leq k_{1}$. Then by Proposition 2.1, we have (b).

Moreover there exist elements $\left\{\mu_{j}\right\}_{k_{1}+k_{2}+1 \leq j \leq k_{1}+k_{2}+2},\left\{\nu_{j}\right\}_{k_{1}+k_{2}+1 \leq j \leq k_{1}+k_{2}+2}$, of $\mathbb{Z}_{p}$ such that

$$
B\left(z_{j}+\mu_{j} z_{1}^{\prime}+\nu_{j} z_{2}^{\prime}, z_{i}^{\prime}\right)=0 \quad\left(k_{1}+k_{2}+1 \leq j \leq k_{1}+k_{2}+2,1 \leq i \leq 2\right) .
$$


Put

$$
z_{j}^{\prime}= \begin{cases}z_{j}+\mu_{j} z_{1}^{\prime}+\nu_{j} z_{2}^{\prime}, & k_{1}+k_{2}+1 \leq j \leq k_{1}+k_{2}+2 \\ z_{j}, & k_{1}+k_{2}+3 \leq j \leq k .\end{cases}
$$
Then we can easily see that the statement (c),(d), and (e) are satisfied by such $z_{j}^{\prime}$ 's.
This completes the proof. $\quad \square$

By applying Lemma 2.2, we have the following proposition.

Proposition 2.3. Let $m_{1}, m_{2}$ and $n$ be non-negative integers such that $m=m_{1}+$ $m_{2}$. Let $K=\mathbb{Z}_{p}\left[z_{1}, \ldots, z_{m+n}\right]$ and $W=\mathbb{Z}_{p}\left[w_{1}, \ldots, w_{n}\right]$ be quadratic modules over $\mathbb{Z}_{p}$ such that

$$
\left(B\left(z_{i}, z_{j}\right)\right)_{1 \leq i, j \leq m+n}=A \perp B, \quad\left(B\left(w_{i}, w_{j}\right)\right)_{1 \leq i, j \leq n}=O_{n},
$$

where $A=V \perp U$ is unimodular and a canonical form, $\operatorname{deg}(V)=m_{1}, \operatorname{deg}(U)=m_{2}$ and $B=\left(b_{i j}\right)$ is a non-degenerate matrix of degree $n$ and assume that $b_{11} \in$ $4 p \mathbb{Z}_{p}$. Put $M_{1}=\mathbb{Z}_{p}\left[z_{1}, \ldots, z_{m_{1}}\right], M_{2}=\mathbb{Z}_{p}\left[z_{m_{1}+1}, \ldots, z_{m}\right], M=M_{1} \perp M_{2}, N=$ $\mathbb{Z}_{p}\left[z_{m+1}, \ldots z_{m+n}\right]$ and $W_{1}=\mathbb{Z}_{p}\left[w_{1}\right]$. Let $\phi_{1} \in L_{e}\left(W_{1}, K\right)$ such that $\operatorname{Pr}_{K \rightarrow N} \phi_{1}\left(w_{1}\right)=$ $z_{m+1}$.

(1) Let $p \neq 2$. Assume that $\operatorname{Pr}_{K \rightarrow M_{1}} \phi_{1}\left(W_{1}\right) \not \subset p M_{1}$. Then there exist elements $\left\{z_{i}^{\prime}\right\}_{3 \leq i \leq m+n}$ of $K$ such that

$$
\left(B\left(z_{i}^{\prime}, z_{j}^{\prime}\right)\right)_{3 \leq i, j \leq m+n}=A^{(1)} \perp B
$$

and

$$
\# L_{e}\left(W, K ; M, N ; \mathbf{w}, \mathbf{v} ; W_{1}, \phi_{1}\right)=\# L_{e}\left(\hat{W}, K^{\prime} ; M^{\prime}, N^{\prime} ; \mathbf{w}^{\prime}, \mathbf{v}^{\prime}\right),
$$

where $\hat{W}=\mathbb{Z}_{p}\left[w_{2}, \ldots, w_{n}\right], M^{\prime}=\mathbb{Z}_{p}\left[z_{3}^{\prime}, \ldots, z_{m+1}^{\prime}\right], N^{\prime}=\mathbb{Z}_{p}\left[z_{m+2}^{\prime}, \ldots, z_{m+n}^{\prime}\right], \mathbf{w}$ ' $\left\{w_{2}, \ldots, w_{n}\right\}$ and $\mathbf{v}^{\prime}=\left\{z_{m+2}^{\prime}, \ldots, z_{m+n}^{\prime}\right\}$.

(2) Let $p=2$. Assume that $\operatorname{Pr}_{K \rightarrow M_{1}} \phi_{1}\left(W_{1}\right) \not \subset p M_{1}$ or $U \neq 1 \perp-1$. Then there exist elements $\left\{z_{i}^{\prime}\right\}_{3 \leq i \leq m+n}$ of $K$ such that

$$
\left(B\left(z_{i}^{\prime}, z_{j}^{\prime}\right)\right)_{3 \leq i, j \leq m+n}=A^{(1)} \perp B
$$

and

$$
\# L_{e}\left(W, K ; M, N ; \mathbf{w}, \mathbf{v} ; W_{1}, \phi_{1}\right)=\# L_{e}\left(\hat{W}, K^{\prime} ; M^{\prime}, N^{\prime} ; \mathbf{w}^{\prime}, \mathbf{v}^{\prime}\right),
$$

where the notations are the same as in (1).

(3) Let $p=2$. Assume that $\operatorname{Pr}_{K \rightarrow M} \phi_{1}\left(W_{1}\right) \not \subset p M$ and $\operatorname{Pr}_{K \rightarrow M_{1}} \phi_{1}\left(W_{1}\right) \subset p M_{1}$ and $U=1 \perp-1$, then there exist elements $\left\{z_{i}^{\prime}\right\}_{3 \leq i \leq m+n}$ of $K$ such that

$$
\left(B\left(z_{i}^{\prime}, z_{j}^{\prime}\right)\right)_{3 \leq i, j \leq m+n}=A^{*} \perp B
$$

and

$$
\# L_{e}\left(W, K ; M, N ; \mathbf{w}, \mathbf{v} ; W_{1}, \phi_{1}\right)=\# L_{e}\left(\hat{W}, K^{\prime} ; M^{\prime}, N^{\prime} ; \mathbf{w}^{\prime}, \mathbf{v}^{\prime}\right),
$$

where the notations are the same as in (1).

Proof. The statement (1) is treated in Katsurada and the present author in [6]. (2) is a slight modification of (1), therefore we shall prove only (3). 
Put $z_{1}^{\prime}=\phi_{1}\left(w_{1}\right)$. Then we have

$$
B\left(z_{1}^{\prime}, z_{1}^{\prime}\right)=0 \bmod p^{e} .
$$

Put $K_{i}=M_{i}$ for $i=1,2$ and $K_{3}=N$. Then for the modules $K_{1}, \ldots, K_{3}$, and the elements $z_{1}^{\prime},\left\{z_{i}\right\}_{1 \leq i \leq m+n}$ stated above, there exist elements $\left\{z_{i}^{\prime}\right\}_{2 \leq i \leq m+n}$ satisfying the conditions $(\mathrm{a}) \sim(\mathrm{e})$ of Lemma $2.2(2)$. Let

$$
z_{j}^{\prime}=\sum_{i=1}^{m+n} \xi_{i j} z_{i}(1 \leq j \leq m+n) \text { with }\left(\xi_{i j}\right) \in G L_{m+n}\left(\mathbb{Z}_{p}\right) .
$$

Then by (a),(d) and (e) of Lemma $2.2(2)$, the matrix $\left(\xi_{i j}\right)_{1 \leq i, j \leq m+n}$ can be expressed as

$$
\left(\xi_{i j}\right)=\left(\begin{array}{ccc}
\Xi_{11} & \Xi_{12} & \Xi_{13} \\
\Xi_{21} & 0_{n-1, m} & \Xi_{23}
\end{array}\right)
$$

where $\Xi_{23} \equiv E_{n-1} \bmod 2$.

The assumption that $\operatorname{Pr}_{K \rightarrow N} \phi_{1}\left(w_{1}\right)=z_{m+1}$ implies

$$
\Xi_{21}=\left(\begin{array}{c}
1 \\
0_{n-1,1}
\end{array}\right) \text {. }
$$

Then by $(\mathrm{a}) \sim(\mathrm{e})$ of Lemma $2.2(2)$, we have

$$
\left(B\left(z_{i}^{\prime}, z_{j}^{\prime}\right)\right)_{3 \leq i, j \leq m+n}=A^{*} \perp B .
$$

Thus, it suffices to prove that there exists a bijection of $L_{e}\left(W, K ; M, N ; \mathbf{w}, \mathbf{v} ; W_{1}, \phi_{1}\right)$ to $L_{e}\left(\hat{W}, K^{\prime} ; M^{\prime}, N^{\prime} ; \mathbf{w}^{\prime}, \mathbf{v}^{\prime}\right)$. To prove this for an element $\phi^{\prime}$ of $\operatorname{Hom}_{\mathbb{Z}_{p}}\left(\hat{W}, K^{\prime}\right)$ by $\phi^{\prime}=\operatorname{Pr}_{K \rightarrow K^{\prime}} \phi \mid \hat{W}$. Then $\phi^{\prime}$ belongs to $L_{e}\left(\hat{W}, K^{\prime}\right)$. In fact for $1 \leq j \leq n$

$$
\phi\left(w_{j}\right)=\sum_{i=1}^{m+n} x_{i j} z_{i} \text { with }\left(x_{i j}\right) \in M_{m+n, n}\left(\mathbb{Z}_{p}\right) .
$$

Then for $1 \leq j \leq n$ we have

$$
\phi\left(w_{j}\right)=\sum_{i=1}^{m+n} x_{i j}^{\prime} z_{i}^{\prime}
$$

with

$$
x_{i j}=\sum_{a=1}^{m+n} \xi_{i a} x_{a j}^{\prime} .
$$

Since $\phi\left(w_{1}\right) \equiv z_{1}^{\prime} \bmod p^{e}$ and $\phi^{\prime}\left(w_{j}\right)=\phi\left(w_{j}\right)-x_{1 j}^{\prime} z_{1}^{\prime}-x_{2 j}^{\prime} z_{2}^{\prime}(2 \leq j \leq n)$, we have $B\left(\phi\left(w_{1}\right), \phi\left(w_{j}\right)\right) \equiv B\left(z_{1}^{\prime}, x_{1 j}^{\prime} z_{1}^{\prime}+x_{2 j}^{\prime} z_{2}^{\prime}\right) \equiv 0 \bmod p^{e}$ for $2 \leq j \leq n$. Thus by $(2.3)$ and (a) of Lemma 2.2 (1) we have $x_{2 j}^{\prime} \equiv 0 \bmod p^{e}$ for $2 \leq j \leq n$. Thus we have

$$
B\left(\phi^{\prime}\left(w_{i}\right), \phi^{\prime}\left(w_{j}\right)\right) \equiv B\left(\phi\left(w_{i}\right), \phi\left(w_{j}\right)\right) \bmod p^{e} \text { for any } 2 \leq i, j \leq n \text {. }
$$


Since $B\left(\phi\left(w_{i}\right), \phi\left(w_{j}\right)\right) \equiv B\left(w_{i}, w_{j}\right) \bmod p^{e}$ for any $2 \leq i, j \leq n, \phi^{\prime}$ belongs to $L_{e}\left(\hat{W}, K^{\prime}\right)$. Furthermore by $(2.3),(2.4)$ and $(2.7)$, we have $x_{i j} \equiv x_{i j}^{\prime} \bmod p^{e}(m+$ $2 \leq i \leq m+n, 2 \leq j \leq n)$. Thus we have for $1 \leq i \leq n-1,1 \leq j \leq n-1$,

$$
\operatorname{Pr}_{K \rightarrow N} \phi\left(w_{j}\right)=\delta_{i j} z_{m+i} \text { if and only if } \operatorname{Pr}_{K^{\prime} \rightarrow N^{\prime}} \phi^{\prime}\left(w_{j+1}\right)=\delta_{i j} z_{m+i+1}^{\prime} \text {. }
$$

Thus $\phi^{\prime}$ belongs to $L_{e}\left(\hat{W}, K^{\prime} ; M^{\prime}, N^{\prime} ; \mathbf{w}^{\prime}, \mathbf{v}^{\prime}\right)$. Thus we can define a mapping $\pi$ from $L_{e}\left(W, K ; M, N ; \mathbf{w}, \mathbf{v} ; W_{1}, \phi_{1}\right)$ to $L_{e}\left(\hat{W}, K^{\prime} ; M^{\prime}, N^{\prime} ; \mathbf{w}^{\prime}, \mathbf{v}^{\prime}\right)$ by

$$
\pi(\phi)=\phi^{\prime} .
$$

Let $\phi$ be an element of $L_{e}\left(W, K ; M, N ; \mathbf{w}, \mathbf{v} ; W_{1}, \phi_{1}\right)$. Then by (2.2), (2.4) and (2.7), we have

$$
x_{m+1, j}=x_{1 j}^{\prime}+\xi_{m+1, m+1} x_{m+1, j}^{\prime}+\xi_{m+1, m+2} x_{m+2, j}^{\prime} .
$$

Then we have for $2 \leq j \leq n$

$\operatorname{Pr}_{K \rightarrow N}\left(\phi\left(w_{j}\right)\right)=\delta_{1 j} z_{m+1}$ if and only if $x_{1 j}^{\prime} \equiv-\xi_{m+1, m+1} x_{m+1, j}^{\prime}-\xi_{m+1, m+2} x_{m+2, j}^{\prime} \bmod p^{e}$, this shows the injectivity of $\pi$.

Lastly we prove $\pi$ is surjective. Take an element $\phi^{\prime}$ in $L_{e}\left(\hat{W}, K^{\prime} ; M^{\prime}, N^{\prime} ; \mathbf{w}^{\prime}, \mathbf{v}^{\prime}\right)$ and put

$$
\phi^{\prime}\left(w_{j}\right)=\sum_{i=3}^{m+n} y_{i j}^{\prime} z_{i}^{\prime}(2 \leq j \leq n) .
$$

Put $y_{1 j}^{\prime}=-\xi_{m+1, m+1} y_{m+1, j}^{\prime}-\xi_{m+1, m+2} y_{m+2, j}^{\prime}$ and define a mapping $\phi$ from $W$ to $K$ by

$$
\phi\left(w_{1}^{\prime}\right)=z_{1}^{\prime}, \phi\left(w_{j}\right)=-y_{1 j}^{\prime} z_{1}^{\prime}+\sum_{i=3}^{m+n} y_{i j}^{\prime} z_{i}^{\prime}(2 \leq j \leq n) .
$$

Then by construction and $(2.8),(2.9)$ and $(2.10)$, we have

$$
\phi \in L_{e}\left(W, K ; M, N ; \mathbf{w}, \mathbf{v} ; W_{1}, \phi_{1}\right) \text { and } \pi(\phi)=\phi^{\prime} .
$$

Thus we complete the proof.

Remark. (1) Under the above notaion we see easily that

$$
\# L_{e}\left(\hat{W}, K^{\prime} ; M^{\prime}, N^{\prime} ; \mathbf{w}^{\prime}, \mathbf{v}^{\prime}\right)=\# A_{e}\left(n-1,-B, A^{(1)}\right)
$$

or

$$
\# L_{e}\left(\hat{W}, K^{\prime} ; M^{\prime}, N^{\prime} ; \mathbf{w}^{\prime}, \mathbf{v}^{\prime}\right)=\# A_{e}\left(n-1,-B, A^{*}\right)
$$

according as in (1), (2) or in (3).

(2) In the above proposition, assume that $N=N_{1} \perp \hat{N}$, where $N_{1}$ and $\hat{N}$ are quadratic submodules of rank 1 and $n-1$, respectively, and $M=<A>$ is unimodular and $\phi_{1} \in L_{e}\left(N_{1}^{\prime}, M\right)$, where for a quadratic module $L=\langle S\rangle$ we write $L^{\prime}=\langle-S\rangle$. Then by Proposition 1.3 and Prorosition 2.3 we have

$\# L_{e}\left(N^{\prime}, M ; \phi_{1}\right)=\# L_{e}\left(\hat{N}^{\prime}, M^{(1)} \perp N_{1}\right)$ or $\# L_{e}\left(N^{\prime}, M ; \phi_{1}\right)=\# L_{e}\left(\hat{N}^{\prime}, M^{*} \perp N_{1}\right)$ where $M^{(1)}=<A^{(1)}>$ and $M^{*}=<A^{*}>$ according as the case (1),(2) or (3) in Proposition 2.3. A part of the former case is nothing but [2, Proposition 2.2 (1.b)].

Summarizing Proposition 1.1 and Proposition 2.3, we have the following proposition. 
Proposition 2.4.. Let $A=V \perp U$ and $B$ be as the same in Proposition 2.3. Let $\Xi={ }^{t}\left(\Xi_{1} \quad \Xi_{2} \quad \Xi_{3}\right) \in A_{e}\left(b_{11}, A\right)$, where $\Xi_{1} \in\left(\mathbb{Z}_{p} / p^{e} \mathbb{Z}_{p}\right)^{m_{1}}, \Xi_{2} \in\left(\mathbb{Z}_{p} / p^{e} \mathbb{Z}_{p}\right)^{m_{2}}$ and $e \geq 2$. Assume that $\left(\begin{array}{l}\Xi_{1} \\ \Xi_{2}\end{array}\right) \not \equiv 0 \bmod p$.

(1) Let $p \neq 2$. Assume that $\Xi_{1} \not \equiv 0 \bmod p$. Then we have

$$
a_{e}(B, A ; \Xi)=a_{e}\left(n-1 ; B, A^{(1)}\right) .
$$

(2) Let $p=2$. Assume that $\Xi_{1} \not \equiv 0 \bmod p$ or $U \neq 1 \perp-1$. Then we have

$$
a_{e}(B, A ; \Xi)=a_{e}\left(n-1 ; B, A^{(1)}\right) .
$$

(3) Let $p=2$. Assume that $\Xi_{1} \equiv 0 \bmod p$ and $U=1 \perp-1$. Then we have

$$
a_{e}(B, A ; \Xi)=a_{e}\left(n-1 ; B, A^{*}\right) .
$$

Remark. Assume that the level of $\hat{T} \in S_{t-1}\left(\mathbb{Z}_{p}\right)$ is equal to or smaller than $\nu\left(2^{-1} b_{1}\right)-1$. Then the matrix $\hat{T}+b_{1}\left(y_{i} y_{j}\right)_{1 \leq i, j \leq t-1}$ is equivalent to $\hat{T}$ for any $\left(y_{i}\right) \in \mathbb{Z}_{p}^{t-1}$. Thus for any symmetric matrix $S$ we have

$$
\begin{aligned}
a_{e}\left(t-1 ; b_{1} \perp \hat{T}, S\right) & =\sum_{\left(y_{i}\right) \in\left(\mathbb{Z}_{p} / p^{c} \mathbb{Z}_{p}\right)^{t-1}} a_{e}\left(\hat{T}+b_{1}\left(y_{i} y_{j}\right)_{1 \leq i, j \leq t-1}, S\right) \\
& =p^{e(t-1)} a_{e}(\hat{T}, S) .
\end{aligned}
$$

Thus we have

$$
\alpha_{p}\left(t-1 ; b_{1} \perp \hat{T}, S\right)=\alpha_{p}(\hat{T}, S)
$$

\section{A recursion formula}

For $S \in S_{m}\left(\mathbb{Z}_{2}\right), T \in S_{n}\left(\mathbb{Z}_{2}\right), \bar{\Theta}=\left(\bar{\theta}_{i}\right) \in\left(\mathbb{Z}_{p} / p \mathbb{Z}_{p}\right)^{m}$, and a non-negative integer $e$, put (cf. [3])

$$
\bar{A}_{e}(T, S ; \bar{\Theta})=\left\{\left(x_{i j}\right) \in A_{e}(T, S) ; x_{i 1} \equiv \theta_{i} \bmod 2 \text { for any } 1 \leq i \leq m\right\},
$$

and

We note that

$$
\bar{a}_{e}(T, S ; \bar{\Theta})=\# \bar{A}_{e}(T, S ; \bar{\Theta}) .
$$

$$
\bar{a}_{e}(T, S ; \bar{\Theta})=\sum_{\Xi} a_{e}(T, S ; \Xi),
$$

where $\Xi$ runs through all representatives of $\left(\mathbb{Z}_{p} / p^{e} \mathbb{Z}_{p}\right)^{m}$ such that $\Xi \equiv \Theta \bmod p$.

For symmetric matrices $A$ and $B$ of degree $m$ and $n(m \geq n \geq 1)$, respectively, with entries in the ring $\mathbb{Z}_{p}$ of $p$-adic integers, define a primitive local density $\beta_{p}(T, S)$ by

$$
\beta_{p}(B, A)=\lim _{e \rightarrow \infty} p^{-t e} \# B_{e}(B, A),
$$

where $t=m n-n(n+1) / 2$ and

$$
B_{e}(B, A)=\left\{X \in A_{e}(B, A) ; X \text { is primitive }\right\} .
$$

Here $X$ is said to be primitive if its reduction modulo $p$ has the maximal rank. 
Lemma 3.1. Let $A=V \perp U \in S_{m}\left(\mathbb{Z}_{2}\right) \cap G L_{m}\left(\mathbb{Z}_{2}\right)$ be a unimodular matrix such that $V$ (resp. $U$ ) is an even unimodular (resp. odd unimodular) part of degree $m_{1}$ (resp. $m_{2}$ ). Let $\bar{\Theta}_{1} \in\left(\mathbb{Z}_{2} / 2 \mathbb{Z}_{2}\right)^{m_{1}}, \bar{\Theta}_{2} \in\left(\mathbb{Z}_{2} / 2 \mathbb{Z}_{2}\right)^{m_{2}}$ and $b \in 2 \mathbb{Z}_{2}$. have

(1) Assume that $e \geq 2, \Theta_{1} \not \equiv 0_{m_{1}, 1} \bmod 2, \Theta_{2} \equiv 0_{m_{2}, 1}$ or $1_{m_{2}, 1} \bmod 2$. Then we

$$
\begin{aligned}
& 2^{(-m+1) e} \bar{a}_{e}\left(b, A ;\left(\begin{array}{c}
\bar{\Theta}_{1} \\
\bar{\Theta}_{2}
\end{array}\right)\right) \\
& =\left\{\begin{array}{ll}
2^{-m+2}, & \text { either if } \Theta_{2} \equiv 0_{m_{2}, 1} \bmod 2 \text { and } V\left[\Theta_{1}\right] \equiv b \bmod 4 \\
0, & \text { or if } \Theta_{2} \equiv 1_{m_{2}, 1} \bmod 2 \text { and } V\left[\Theta_{1}\right] \equiv-\operatorname{tr}(U)+b \bmod 4,
\end{array},\right.
\end{aligned}
$$

(2) Assume that $e \geq 3, U=\left(\begin{array}{cc}1 & 0 \\ 0 & -1\end{array}\right), \Theta_{1} \equiv 0_{m_{1}, 1} \bmod 2$. Then we have

$$
2^{(-m+1) e} \bar{a}_{e}(0, A ; \bar{\Theta})=\left\{\begin{array}{l}
2^{-m+3}, \text { if } U\left[\Theta_{2}\right] \equiv 0 \bmod 8 \\
0, \text { otherwise }
\end{array}\right.
$$

Proof. For $e \geq 1$, put

$$
\overline{\mathcal{A}}_{e}^{\prime}(\Theta)=\left\{\bar{X}=\left(\bar{x}_{i 1}\right) \in\left(\mathbb{Z}_{2} / 2^{e} \mathbb{Z}_{2}\right)^{m} ; A[X] \equiv b \bmod 2^{e+1}, x_{i 1} \equiv \theta_{i} \bmod 2\right\} .
$$

For $e \geq 2$, consider the map $\Phi: \bar{A}_{e}(b, A ; \bar{\Theta}) \rightarrow \overline{\mathcal{A}}_{e-1}^{\prime}(\Theta)$ defined by $X \bmod$ $2^{e} \mapsto X \bmod 2^{e-1}$ for $e \geq 2$. It is a surjection, and we have $\# \Phi^{-1}(\bar{X})=2^{m}$ for any $\bar{X} \in \overline{\mathcal{A}}_{e-1}^{\prime}(\Theta)$. Next consider the map $\Psi: \overline{\mathcal{A}}_{e}^{\prime}(\Theta) \rightarrow \overline{\mathcal{A}}_{e-1}^{\prime}(\Theta)$ defined by $X$ $\bmod 2^{e} \mapsto X \bmod 2^{e-1}$. Obviously it is a surjection.

(1) It is easy to see that if $\Theta_{2} \not \equiv 0_{m_{2}, 1}, 1_{m_{2}, 1} \bmod 2$, then

$$
\bar{a}_{e}(b, A ; \bar{\Theta})=0 \text {. }
$$

First assume that $\Theta_{2} \equiv 0_{m_{2}, 1} \bmod 2$. For any $\bar{Y}=\left(\begin{array}{c}\bar{Y}_{1} \\ \bar{Y}_{2}\end{array}\right) \in \overline{\mathcal{A}}_{e-1}^{\prime}(\Theta)$, put $\bar{X}=\bar{Y}+2^{e-1} \bar{Z} \in \overline{\mathcal{A}}^{\prime}{ }_{e}(\Theta), \bar{Z}=\left(\begin{array}{c}\bar{Z}_{1} \\ \bar{Z}_{2}\end{array}\right) \in\left(\mathbb{Z}_{2} / 2 \mathbb{Z}_{2}\right)^{m}$. Then

$$
A[X] \equiv A[Y]+2^{e} \cdot{ }^{t} Z_{1} A^{*} Y_{1} \equiv b \quad \bmod 2^{e+1} .
$$

Since $\Theta_{1} \not \equiv 0_{m_{1}, 1} \bmod 2$, we have $A^{*} Y_{1} \equiv A^{*} \theta_{1} \not \equiv 0_{m_{1}, 1} \bmod 2$, and $\#\left\{Z_{1} \in\right.$ $\left.\left(\mathbb{Z}_{2} / 2 \mathbb{Z}_{2}\right)^{m_{1}} ;{ }^{t} Z_{1} A^{*} Y_{1} \equiv 2^{-e}(b-A[Y]) \bmod 2\right\}=2^{m_{1}-1}$. Thus $\Psi^{-1}(\bar{Y})=2^{m-1}$. Moreover $\# \overline{\mathcal{A}}_{1}^{\prime}(\Theta)=1$. After all, we obtain that

$$
\begin{aligned}
\bar{a}_{e}(b, A ; \bar{\Theta}) & =2^{m} \# \overline{\mathcal{A}}_{e-1}^{\prime}(\Theta)=\cdots=2^{m} 2^{(e-2)(m-1)} \# \overline{\mathcal{A}}_{1}^{\prime}(\Theta) \\
& =2^{m} 2^{(e-2)(m-1)}
\end{aligned}
$$

for $e \geq 2$.

By similar argument as above we get $\bar{a}_{e}(b, A ; \bar{\Theta})=2^{m} 2^{(e-2)(m+1)}$ for $e \geq 2$ when $\Theta_{2} \equiv 1_{m_{2}, 1} \bmod 2$. 
(2) Similar argument implies

$$
\bar{a}_{e}(0, A ; \bar{\Theta})=2^{m} \# \overline{\mathcal{A}}_{e-1}^{\prime}(\Theta)=\cdots=2^{m} 2^{(e-3)(m-1)} \# \overline{\mathcal{A}}_{2}^{\prime}(\Theta) .
$$

Since

$$
\begin{gathered}
\# \overline{\mathcal{A}}_{2}^{\prime}(\Theta)=\#\left\{\bar{X} \in\left(\mathbb{Z}_{2} / 4 \mathbb{Z}_{2}\right)^{m} ; A^{*}\left[X_{1}\right]+V\left[X_{2}\right] \equiv 0 \bmod 8,\right. \\
\left.X_{1} \equiv 0 \bmod 2, X_{2} \equiv 1 \bmod 2\right\} \\
=2^{m-2} \#\left\{\left(\bar{x}_{i}\right) \in\left(\mathbb{Z}_{2} / 4 \mathbb{Z}_{2}\right)^{2} ; x_{1}^{2}-x_{2}^{2} \equiv 0 \bmod 8, x_{1} \equiv x_{2} \equiv 1 \bmod 2\right\},
\end{gathered}
$$

we have $\overline{\mathcal{A}}_{2}^{\prime}(\Theta)=2^{m}$, this completes the proof.

We shall make a distinction of the following types as above.

(case I) $p \neq 2$,

(case II) $p=2$ and $U \neq 1 \perp-1$,

(case III) $p=2$ and $U=1 \perp-1$.

The next theorem in the case for $p \neq 2$ and that $B$ is diagonal is given in [3] and in the case that $A$ is even unimodular is given in [6],[7]. We get here the generalized formula without such restrictions such that we shall prove the odd unimodular case.

Theorem 3.2. Let $A=A^{*} \perp U$ be a symmetric unimodular matrix of degree $m$ and $B$ be a non-degenerate matrix of degree $n$ as above. Then we have

$$
\begin{aligned}
& \alpha_{p}(B, A)-p^{-m+n+1} \alpha_{p}\left(B\left[p^{-1} \perp E_{n-1}\right], A\right) \\
& \quad= \begin{cases}\beta_{p}(0, A) \alpha_{p}\left(n-1 ; B, A^{(1)}\right), & \text { (case I) }, \\
p^{-2}\left\{\beta_{p}\left(0, A^{*}\right)+\beta_{p}\left(t r(-U), A^{*}\right)\right\} \alpha_{p}\left(n-1 ; B, A^{(1)}\right), & \text { (case II), } \\
p^{-1} \beta_{p}\left(0, A^{*}\right) \alpha_{p}\left(n-1 ; B, A^{(1)}\right)+p^{-m+3} \alpha_{p}\left(n-1 ; B, A^{*}\right), & \text { (case III), }\end{cases}
\end{aligned}
$$

where we make the convention that $\alpha_{p}\left(n-1 ; B, A^{(1)}\right)=0$ if the Witt index of $A$ is 0 and $\beta_{p}\left(\operatorname{tr}(-U), A^{*}\right)=0$ if $U=\varnothing$.

Proof. The proof in the case $A$ is even unimodular is completed in [6] and [7], so we shall prove the case that $A$ is odd unimodular and $p=2$.

We assume that $e \geq 3$. Then we have

$$
a_{e}(B, A)=\sum_{\bar{\Theta} \in\left(\mathbb{Z}_{2} / 2 \mathbb{Z}_{2}\right)^{m}} \bar{a}_{e}(B, A ; \bar{\Theta}) .
$$

Let $\operatorname{deg}\left(A^{*}\right)=m_{1}$ and $\operatorname{deg}(U)=m_{2}$.

Put for $a \in \mathbb{Z}_{2}$,

$$
\mathcal{S}(a)=\left\{\bar{\Theta} \in\left(\mathbb{Z}_{2} / 2 \mathbb{Z}_{2}\right)^{m_{1}} ; \Theta \not \equiv 0_{m_{1}, 1} \bmod 2, A^{*}[\Theta] \equiv a \bmod 4\right\}
$$

Further put

$$
\mathcal{S}=\left\{\bar{\Theta} \in\left(\mathbb{Z}_{2} / 2 \mathbb{Z}_{2}\right)^{m_{2}} ; U[\Theta] \equiv 0_{m_{2}, 1} \bmod 8, \Theta \not \equiv 0 \bmod 2\right\}
$$


and

$$
\begin{gathered}
\mathcal{S}^{\prime}=\left\{\left(\bar{\Theta}_{1}, \bar{\Theta}_{2}\right) \in\left(\mathbb{Z}_{2} / 2 \mathbb{Z}_{2}\right)^{m_{1}} \times\left(\mathbb{Z}_{2} / 2 \mathbb{Z}_{2}\right)^{m_{2}} ; A^{*}\left[\Theta_{1}\right]+U\left[\Theta_{2}\right] \equiv 0 \bmod 4,\right. \\
\left.\Theta_{1} \neq 0_{2 d, 1} \bmod 2, \Theta_{2} \not \equiv 0_{m_{2}, 1} \bmod 2\right\} .
\end{gathered}
$$

Then we have

$$
\begin{aligned}
a_{e}(B, A) & =\bar{a}_{e}\left(B, A ; \overline{0}_{m 1}\right) \\
& +\sum_{\bar{\Theta}_{1} \in \mathcal{S}(0)} \bar{a}_{e}\left(B, A ;\left(\begin{array}{c}
\bar{\Theta}_{1} \\
\overline{0}_{m-2 d, 1}
\end{array}\right)\right) \\
& +\sum_{\bar{\Theta}_{2} \in \mathcal{S}} \bar{a}_{e}\left(B, A ;\left(\begin{array}{c}
\overline{0}_{2 d, 1} \\
\bar{\Theta}_{2}
\end{array}\right)\right) \\
& +\sum_{\left(\bar{\Theta}_{1}, \bar{\Theta}_{2}\right) \in \mathcal{S}^{\prime}} \bar{a}_{e}\left(B, A ;\left(\begin{array}{c}
\bar{\Theta}_{1} \\
\bar{\Theta}_{2}
\end{array}\right)\right) .
\end{aligned}
$$

(case II) Since $U \neq\left(\begin{array}{cc}1 & 0 \\ 0 & -1\end{array}\right)$, we have $\mathcal{S}=\varnothing$. If $\operatorname{deg}(U)=1$ then it is to see that $\mathcal{S}^{\prime}=\varnothing$. Next we assume $\operatorname{deg}(U)=2$. If $\left(\bar{\Theta}_{1}, \bar{\Theta}_{2}\right) \in \mathcal{S}^{\prime}$, then $\bar{\Theta}_{2}=\overline{1}_{21}$. In fact, since $A^{*}\left[\Theta_{1}\right] \equiv 0 \bmod 2$ for any $\bar{\Theta}_{1} \in\left(\mathbb{Z}_{2} / 2 \mathbb{Z}_{2}\right)^{m_{1}}$, so that we have $U\left[\Theta_{2}\right] \equiv 0 \bmod 2$, therefore $\bar{\Theta}_{2}=\overline{1}_{21}$. Then $U\left[\Theta_{2}\right] \equiv \operatorname{tr}(U) \bmod 4$, and we have

$$
\begin{aligned}
a_{e}(B, A) & =\bar{a}_{e}\left(B, A ; \overline{0}_{m 1}\right) \\
& +\sum_{\bar{\Theta}_{1} \in \mathcal{S}(0)} \bar{a}_{e}\left(B, A ;\left(\begin{array}{c}
\bar{\Theta}_{1} \\
\overline{0}_{m_{2}, 1}
\end{array}\right)\right) \\
& +\sum_{\Theta_{1} \in \mathcal{S}(-\operatorname{tr}(U))} \bar{a}_{e}\left(B, A ;\left(\begin{array}{c}
\bar{\Theta}_{1} \\
\overline{1}_{m_{2}, 1}
\end{array}\right)\right) .
\end{aligned}
$$

Note that the last term on the right-hand side is 0 if $\operatorname{deg}(U) \neq 2$.

We can show

$$
2^{e(-m n+n(n+1) / 2)} \bar{a}_{e}\left(B, A ; \overline{0}_{m 1}\right)=2^{-m+1} \alpha_{2}\left(B\left[2^{-1} \perp E_{n-1}\right], A\right)
$$

for a sufficiently large $e$ in the same way as in the proof of [3, Proposition 3.6(2)]. Next we show that

$$
\begin{gathered}
2^{e(-m n+n(n+1) / 2)} \sum_{\bar{\Theta}_{1} \in \mathcal{S}(0)} \bar{a}_{e}\left(B, A ;\left(\begin{array}{c}
\bar{\Theta}_{1} \\
\overline{0}_{m_{2}, 1}
\end{array}\right)\right) \\
=\beta_{2}\left(0, A^{*}\right) \alpha_{2}\left(n-1 ; B, A^{(1)}\right)
\end{gathered}
$$

for a sufficient large e. By (2) of Proposition 2.4, for any $\Xi \in \mathbb{Z}_{2}^{m}$ such that $\Xi \equiv\left(\begin{array}{c}\Theta_{1} \\ 0_{m_{2}, 1}\end{array}\right) \bmod 2$, we have

$$
a_{e}(B, A ; \Xi)=a_{e}\left(n-1 ; B, A^{(1)}\right) .
$$


Thus we have

$$
\begin{aligned}
& \sum_{\bar{\Theta}_{1} \in \mathcal{S}(0)} \bar{a}_{e}\left(B, A ;\left(\begin{array}{c}
\bar{\Theta}_{1} \\
\overline{0}_{m_{2}, 1}
\end{array}\right)\right) \\
= & \sum_{\bar{\Theta}_{1} \in \mathcal{S}(0)} \sum_{\Xi} a_{e}\left(n-1 ; B, A^{(1)}\right) \\
= & \sum_{\bar{\Theta}_{1} \in \mathcal{S}(0)} \bar{a}_{e}\left(b_{1}, A ;\left(\begin{array}{c}
\bar{\Theta}_{1} \\
\overline{0}_{m_{2}, 1}
\end{array}\right)\right) a_{e}\left(n-1 ; B, A^{(1)}\right),
\end{aligned}
$$

where $\Xi$ runs over elements of $\left(\mathbb{Z}_{2} / 2^{e} \mathbb{Z}_{2}\right)^{m}$ satisfying that $\Xi \equiv\left(\begin{array}{c}\bar{\Theta}_{1} \\ \overline{0}_{m_{2}, 1}\end{array}\right) \bmod 2$. Therefore we have

$$
\begin{aligned}
& 2^{e(-m n+n(n+1) / 2)} \sum_{\bar{\Theta}_{1} \in \mathcal{S}(0)} \bar{a}_{e}\left(B, A ;\left(\begin{array}{c}
\bar{\Theta}_{1} \\
\overline{0}_{m_{2}, 1}
\end{array}\right)\right) \\
& =2^{(-m+1) e} \sum_{\bar{\Theta}_{1} \in \mathcal{S}(0)} \bar{a}_{e}\left(b_{1}, A ;\left(\begin{array}{c}
\bar{\Theta}_{1} \\
\overline{0}_{m_{2}, 1}
\end{array}\right)\right) \\
& \times 2^{e(-(m-1)(n-1)+(n-1) n / 2)} a_{e}\left(n-1 ; B, A^{(1)}\right) .
\end{aligned}
$$

By Lemma 3.1 (1), for any $\bar{\Theta}_{1} \in \mathcal{S}(0)$, we have

$$
2^{(-m+1) e} \bar{a}_{e}\left(b_{1}, A ;\left(\begin{array}{c}
\bar{\Theta}_{1} \\
\overline{0}_{m_{2}, 1}
\end{array}\right)\right)=2^{-m+2} .
$$

Thus we have

$$
\begin{aligned}
& 2^{e(-m n+n(n+1) / 2)} \sum_{\bar{\Theta}_{1} \in \mathcal{S}(0)} \bar{a}_{e}\left(B, A ;\left(\begin{array}{c}
\bar{\Theta}_{1} \\
\overline{0}_{m_{2}, 1}
\end{array}\right)\right) \\
& =2^{-m+2} \# \mathcal{S}(0) 2^{e(-(m-1)(n-1)+(n-1) n / 2)} a_{e}\left(n-1 ; B, A^{(1)}\right) \\
& =2^{-2} \beta_{2}\left(0, A^{*}\right) \alpha_{2}\left(n-1 ; B, A^{(1)}\right)
\end{aligned}
$$

for a sufficiently large $e$, which implies (3.3). Similarly, we have

$$
\begin{gathered}
2^{e(-m n+n(n+1) / 2)} \sum_{\bar{\Theta}_{1} \in \mathcal{S}(-\operatorname{tr}(U))} \bar{a}_{e}\left(B, A ;\left(\begin{array}{c}
\bar{\Theta}_{1} \\
\overline{1}_{m_{2}, 1}
\end{array}\right)\right) \\
=2^{-2} \beta_{2}\left(-\operatorname{tr}(U), A^{*}\right) \alpha_{2}\left(n-1 ; B, A^{(1)}\right),
\end{gathered}
$$

for a sufficiently large $e$. Thus the assertion (case II) follows from (3.2), (3.3), and (3.4). 
Next we prove (case III). We have

$$
\begin{aligned}
a_{e}(B, A) & =\bar{a}_{e}\left(B, A ; \overline{0}_{m 1}\right) \\
& +\sum_{\bar{\Theta}_{1} \in \mathcal{S}(0)}\left\{\bar{a}_{e}\left(B, A ;\left(\begin{array}{c}
\bar{\Theta}_{1} \\
\overline{0}_{21}
\end{array}\right)\right)+\bar{a}_{e}\left(B, A ;\left(\begin{array}{c}
\bar{\Theta}_{1} \\
\overline{1}_{21}
\end{array}\right)\right)\right\} \\
& +\sum_{\bar{\Theta}_{2}} \bar{a}_{e}\left(B, A ;\left(\begin{array}{c}
\overline{0}_{m_{1}, 1} \\
\bar{\Theta}_{2}
\end{array}\right)\right),
\end{aligned}
$$

where $\bar{\Theta}_{2}$ runs over the set

$$
\mathcal{S}^{\prime \prime}=\left\{\bar{\Theta} \in\left(\mathbb{Z}_{2} / 2 \mathbb{Z}_{2}\right)^{2} ;\left(\begin{array}{cc}
1 & 0 \\
0 & -1
\end{array}\right)[\Theta] \equiv 0 \bmod 8, \Theta \not \equiv 0_{21} \bmod 2\right\}
$$

Since $\operatorname{tr}(U)=0$, we see similarly as in (3.4) that

$$
\begin{gathered}
2^{e(-m n+n(n+1) / 2)} \sum_{\bar{\Theta}_{1} \in \mathcal{S}(0)} \bar{a}_{e}\left(B, A ;\left(\begin{array}{l}
\bar{\Theta}_{1} \\
\overline{1}_{21}
\end{array}\right)\right) \\
=2^{-1} \beta_{2}\left(0, A^{*}\right) \alpha_{2}\left(n-1 ; B, A^{(1)}\right)
\end{gathered}
$$

for a sufficiently large $e$. After all, the assertion (case III) reduces to (3.5) below.

$$
2^{e(-m n+n(n+1) / 2)} \sum_{\bar{\Theta}_{2} \in \mathcal{S}^{\prime \prime}} \bar{a}_{e}\left(B, A ;\left(\begin{array}{c}
\overline{0}_{m_{1}, 1} \\
\bar{\Theta}_{2}
\end{array}\right)\right)=2^{-m+3} \alpha_{2}\left(n-1 ; B, A^{*}\right)
$$

for a sufficiently large $e$. By (3) of Proposition 2.4, for any $\Xi \in \mathbb{Z}_{2}^{m}$ such that $\Xi \equiv\left(\begin{array}{c}0_{m_{1}, 1} \\ \Theta_{2}\end{array}\right) \bmod 2$, we have

$$
a_{e}(B, A ; \Xi)=a_{e}\left(n-1 ; B, A^{(1)}\right)
$$

Thus we have

$$
\begin{aligned}
& \sum_{\bar{\Theta}_{2} \in \mathcal{S}^{\prime \prime}} \bar{a}_{e}\left(B, A ;\left(\begin{array}{c}
\overline{0}_{m_{1}, 1} \\
\bar{\Theta}_{2}
\end{array}\right)\right) \\
= & \sum_{\bar{\Theta}_{2} \in \mathcal{S}^{\prime \prime}} \sum_{\Xi} a_{e}\left(n-1 ; B, A^{*}\right) \\
= & \sum_{\bar{\Theta}_{2} \in \mathcal{S}^{\prime \prime}} \bar{a}_{e}\left(b_{1}, A ;\left(\begin{array}{c}
\overline{0}_{m_{1}, 1} \\
\bar{\Theta}_{2}
\end{array}\right)\right) a_{e}\left(n-1 ; B, A^{*}\right),
\end{aligned}
$$

where $\Xi$ runs over elements of $\left(\mathbb{Z}_{2} / 2^{e} \mathbb{Z}_{2}\right)^{m}$ satisfying that $\Xi \equiv\left(\begin{array}{c}0_{m_{1}, 1} \\ \Theta_{2}\end{array}\right) \bmod 2$. Thus we have

$$
\begin{aligned}
& 2^{e(-m n+n(n+1) / 2)} \sum_{\bar{\Theta}_{2} \in \mathcal{S}^{\prime \prime}} \bar{a}_{e}\left(B, A ;\left(\begin{array}{c}
\overline{0}_{m_{1}, 1} \\
\bar{\Theta}_{2}
\end{array}\right)\right) \\
& \left.\left.=2^{(-m+1) e} \sum_{\substack{\bar{\Theta}_{2} \in \mathcal{S}^{\prime \prime} \\
\bar{a}_{e}}} \overline{0}_{m_{1}, 1}\right), A ;\left(\begin{array}{c}
\bar{\Theta}_{2}
\end{array}\right)\right) \\
& \times 2^{e(-(m-1)(n-1)+n(n+1) / 2)} a_{e}\left(n-1 ; B, A^{*}\right) .
\end{aligned}
$$


Then by Lemma $3.1(2)$, for any $\bar{\Theta}_{2} \in \mathcal{S}^{\prime \prime}$, we have

$$
2^{(-m+1) e} \bar{a}_{e}\left(b_{1}, A ;\left(\begin{array}{c}
\overline{0}_{m_{1}, 1} \\
\bar{\Theta}_{2}
\end{array}\right)\right)=2^{-m+3} .
$$

Noting that $\# \mathcal{S}^{\prime \prime}=1$, we have

$$
\begin{aligned}
& 2^{e(-m n+n(n+1) / 2)} \sum_{\bar{\Theta}_{2} \in \mathcal{S}^{\prime \prime}} \bar{a}_{e}\left(B, A ;\left(\begin{array}{c}
\overline{0}_{m_{1}, 1} \\
\bar{\Theta}_{2}
\end{array}\right)\right) \\
& =2^{-m+3} \# \mathcal{S}^{\prime \prime} 2^{e(-(m-1)(n-1)+(n-1) n / 2)} a_{e}\left(n-1 ; B, A^{*}\right) \\
& =2^{-m+3} \alpha_{2}\left(n-1 ; B, A^{*}\right)
\end{aligned}
$$

for a sufficiently large $e$, which proves (3.5) and the assertion (case III).

Remark. It can be easily proved that

$$
\beta_{2}\left(0, A^{*}\right)+\beta_{2}\left(-\operatorname{tr}(U), A^{*}\right)=2^{\operatorname{deg} U} \beta_{2}(0, A) .
$$

Propositon 3.3([6]). Let $e_{0}$ be an integer. Let $S$ and $T$ be non-degenerate symmetric matrices of degree $s, t-1$ respectively, and $T$ be of level $p^{l}$. Assume that $l \geq e_{0}+1-\delta_{2 p}$. Then we have for a sufficiently large $e$

$$
\begin{aligned}
& w\left(1-p^{-s+t-1}\right) p^{e(-s t+t(t+1) / 2)} p^{e_{0}-e} \sum_{a \in \mathbb{Z}_{p} / p^{e-e_{0}} \mathbb{Z}_{p}} \# A_{e}\left(p^{e_{0}} a \perp T, S\right) \\
& =\sum_{a \in \mathbb{Z}_{p}^{\times} /\left(\mathbb{Z}_{p}^{\times}\right)^{2}}\left\{\left(1-p^{-1}-p^{-s+t-1}\right) \alpha_{p}\left(p^{e_{0}} a \perp T, S\right)\right. \\
& \left.\quad+\left(1-p^{-1}\right) p^{-1} \alpha_{p}\left(p^{e_{0}+1} a \perp T, S\right)+p^{-2} \alpha_{p}\left(p^{e_{0}+2} a \perp T, S\right)\right\},
\end{aligned}
$$

where a runs over all elements of $\mathbb{Z}_{p}^{\times} /\left(\mathbb{Z}_{p}^{\times}\right)^{2}$ and $w=\#\left(\mathbb{Z}_{p}^{\times} /\left(\mathbb{Z}_{p}^{\times}\right)^{2}\right)$.

\section{Properties}

In this section we give some concrete formulae for local densities to get an interesting result. Note that the results in this section are treated by Katsurada and the present author in [6].

We set

$$
f(B)= \begin{cases}\beta_{p}(0, A) \alpha_{p}\left(B, A^{(1)}\right), & (\text { case I), } \\ p^{-2}\left\{\beta_{p}\left(0, A^{*}\right)+\beta_{p}\left(\operatorname{tr}(-U), A^{*}\right)\right\} \alpha_{p}\left(B, A^{(1)}\right), & \text { (case II), } \\ p^{-1} \beta_{p}\left(0, A^{*}\right) \alpha_{p}\left(B, A^{(1)}\right)+p^{-m+3} \alpha_{p}\left(B, A^{*}\right), & \text { (case III) } .\end{cases}
$$

First assume that $p \neq 2$. 

Thorem 4.1. Let $A$ be as above. Let $B=p^{r} B_{1} \perp B_{2}$ where $\operatorname{deg} B_{1} \leq 2$ and $p^{s}$ the
level of $B_{2}$.

(1) Let $r \geq s+1$ and $B_{1}=(c)$ with $c \in \mathbb{Z}_{p}^{\times}$. Then we have

$$
\begin{aligned}
\alpha_{p}(B, A) & -p^{-m+n+1} \alpha_{p}\left(B\left[p^{-1} \perp E_{n-1}\right], A\right) \\
& =f\left(B_{2}\right) .
\end{aligned}
$$

(2) Let $r \geq s+1$ and $B_{1}=c_{11} \perp c_{12}$ with $-c_{11} c_{12}^{-1} \notin\left(\mathbb{Z}_{p}^{\times}\right)^{2}$. Then we have

$$
\begin{aligned}
\alpha_{p}(B, A) & -p^{-m+n+1} \alpha_{p}\left(B\left[p^{-1} \perp E_{n-1}\right], A\right) \\
& =p^{-1} \sum_{a=0}^{p-1} f\left(p^{r}\left(c_{11} a^{2}+c_{12}\right) \perp B_{2}\right) .
\end{aligned}
$$

(3) Let $r \geq s$ and $B_{1}=H$. Then we have

$$
\begin{aligned}
\alpha_{p}(B, A) & -p^{-m+n+1} \alpha_{p}\left(B\left[p^{-1} \perp E_{n-1}\right], A\right) \\
& =\frac{1}{2\left(1-p^{-m+n}\right)} \sum_{a}\left\{\left(1-p^{-1}-p^{-m+n}\right) f\left(p^{r} a \perp B_{2}\right)\right. \\
& \left.+\left(1-p^{-1}\right) p^{-1} f\left(p^{r+1} a \perp B_{2}\right)+p^{-2} f\left(p^{r+2} a \perp B_{2}\right)\right\},
\end{aligned}
$$

where a runs over a complete set of representatives of $\mathbb{Z}_{p}^{\times} /\left(\mathbb{Z}_{p}^{\times}\right)^{2}$.

Proof. (1)The assertion can be easily proved by Theorem 3.2 .

(2)By Theorem 3.2, we have

$$
\begin{aligned}
& \alpha_{p}(B, A)-p^{-m+n+1} \alpha_{p}\left(B\left[p^{-1} \perp E_{n-1}\right], A\right) \\
& =\beta_{p}(0, A) \alpha_{p}\left(p^{r} c_{12} \perp B_{2}, A^{(1)} \perp-p^{r} c_{11}\right) .
\end{aligned}
$$

By assumption, $c_{11} y^{2}+c_{12}$ is a unit for any $y \in \mathbb{Z}_{p}$.

Thus we have

$$
\begin{aligned}
& \alpha_{p}\left(p^{r} c_{12} \perp B_{2}, A^{(1)} \perp-p^{r} c_{11}\right) \\
& \quad=p^{-1} \sum_{a=0}^{p-1} \alpha_{p}\left(p^{r}\left(c_{11} a^{2}+c_{12}\right) \perp B_{2}, A^{(1)}\right) .
\end{aligned}
$$

Thus the assertion holds.

(3) For a sufficiently large $e$ we have

$$
\begin{aligned}
& a_{e}\left(n-1 ; B, A^{(1)}\right) \\
& =\sum_{\left(y_{j}\right) \in M_{1, n-1}\left(\mathbb{Z}_{p} / p^{c} \mathbb{Z}_{p}\right)} a_{e}\left(\left(\begin{array}{cc}
2 p^{r} y_{1} & p^{r} \mathbf{y}^{\prime} \\
p^{r} \cdot{ }^{t} \mathbf{y}^{\prime} & B_{2}
\end{array}\right), A^{(1)}\right) \\
& =\sum_{\left(y_{j}\right) \in M_{1, n-1}\left(\mathbb{Z}_{p} / p^{c} \mathbb{Z}_{p}\right)} a_{e}\left(\left(\begin{array}{cc}
2 p^{r} y_{1}-p^{2 r} B_{2}^{-1}\left[{ }^{t} \mathbf{y}^{\prime}\right] & 0 \\
0 & B_{2}
\end{array}\right), A^{(1)}\right),
\end{aligned}
$$


where ${ }^{t} \mathbf{y}^{\prime}=\left(y_{2}, \ldots, y_{n-1}\right)$. By assumption, $2 p^{r} y_{1}-p^{2 r} B_{2}^{-1}\left[{ }^{t} \mathbf{y}^{\prime}\right] \in p^{r} \mathbb{Z}_{p} / p^{e} \mathbb{Z}_{p}$ for any $\left(y_{1}, \ldots, y_{n-1}\right) \in\left(\mathbb{Z}_{p} / p^{e} \mathbb{Z}_{p}\right)^{n-1}$. Thus the map $\Phi$ from $\left(\mathbb{Z}_{p} / p^{e} \mathbb{Z}_{p}\right)^{n-1}$ to $p^{r} \mathbb{Z}_{p} / p^{e} \mathbb{Z}_{p}$ given by $\Phi\left(\left(y_{1}, \ldots, y_{n-1}\right)\right)=2 p^{r} y_{1}-p^{2 r} B_{2}^{-1}\left[{ }^{t} \mathbf{y}^{\prime}\right]$ is a surjection and for any $y \in p^{r} \mathbb{Z}_{p}$, we have $\# \Phi^{-1}(y)=p^{e(n-2)+r}$. Thus we have

$$
a_{e}\left(n-1 ; B, A^{(1)}\right)=p^{e(n-2)+r} \sum_{a \in \mathbb{Z}_{p} / p^{e-r} \mathbb{Z}_{p}} a_{e}\left(p^{r} a \perp B_{2}, A^{(1)}\right) .
$$

Thus the assertion holds by applying Theorem 3.2 and Proposition 3.3.

Corollary 4.2. Let $B_{1}, B_{2}$ and $B_{3}$ be non-degenerate symmetric matrices of level $p^{l_{1}}, p^{l_{2}}$ and $p^{l_{3}}$, respectively, with entries in $\mathbb{Z}_{p}$. Put $B=p^{r} B_{1} \perp p^{r-2} B_{2} \perp B_{3}$. Assume that $B$ is a canonical form, $B_{1}$ is a unimodular matrix of degree $\leq 2, l_{2} \leq 1$ and $r \geq l_{3}+1$. Put $\tilde{B}_{1}=\left(p^{-1} b_{1}\right), p^{-1} b_{1} \perp p b_{2}$ or $H$ according as $B_{1}=\left(b_{1}\right), B_{1}=$ $b_{1} \perp b_{2}$ with $-b_{1} b_{2} \notin\left(\mathbb{Z}_{p}^{\times}\right)^{2}$, or $H$. Then we have

$$
\begin{gathered}
\alpha_{p}(B, A)-p^{-m+n+1} \alpha_{p}\left(p^{r-1} \tilde{B}_{1} \perp p^{r-2} B_{2} \perp B_{3}, A\right) \\
=\sum_{B^{\prime}} c\left(B^{\prime} ; B_{1}, B_{2}\right) f\left(p^{r-2} B^{\prime} \perp B_{3}\right),
\end{gathered}
$$

where where $B^{\prime}$ runs over a certain finitely many symmetric matrices of degree $\operatorname{deg} B_{1}+\operatorname{deg} B_{2}-1$, and $c\left(B^{\prime} ; B_{1}, B_{2}\right)$ is a rational number determined by $B_{1}, B_{2}$ and $A$ such that

$$
\sum_{B^{\prime}} c\left(B^{\prime} ; B_{1}, B_{2}\right)=1
$$

Next we consider the case for $p=2$. We have only to analize $\alpha_{2}\left(n-1 ; B, A^{(1)}\right)$ since the same argument holds for $\alpha_{2}\left(n-1 ; B, A^{*}\right)$ without any modification. Moreover, if $\operatorname{deg}(B)=1$, then a recursion formula is obtained easily so we assume assume $\operatorname{deg}(B)>1$.

Theorem 4.3. Let $B=\perp_{i=0}^{r} 2^{i}\left(U_{i} \perp V_{i}\right)$ be a canonical form, and $\operatorname{deg} U_{i}=k_{i}$, as above.

(1) Assume that $U_{r}=\left(c_{r}\right)$ and $V_{r}=\varnothing$. Put

$$
B_{r-1}=2 U_{r-1} \perp U_{r-2} \perp 2 V_{r-1},
$$

and

$$
B_{r-2}=\perp_{i=0}^{r-3} 2^{i} U_{i} \perp \perp_{i=0}^{r-2} 2^{i} V_{i}
$$

Then we have

$$
\begin{gathered}
\alpha_{2}(B, A)-2^{-m+n+1} \alpha_{2}\left(2^{r-2} c_{r} \perp 2^{r-2} B_{r-1} \perp B_{r-2}, A\right) \\
=8^{-l_{r-1}-k_{r-1}-k_{r-2}} \sum_{\left(y_{i}\right)} f\left(2^{r-2} B_{r-1}\left(\left(y_{i}\right)\right) \perp B_{r-2}\right),
\end{gathered}
$$

where $\left(y_{i}\right)$ runs over all elements of $\left(\mathbb{Z}_{2} / 8 \mathbb{Z}_{2}\right)^{l_{r}+k_{r-1}+k_{r-2}}$ and $B_{r-1}\left(\left(y_{i}\right)\right)$ is a certain symmetric matrix of degree $l_{r}+k_{r-1}+k_{r-2}$ with entries in $\mathbb{Z}_{2}$ determined by $B_{r-1}$ and $\left(y_{i}\right)$ such that $\nu\left(\operatorname{det} B_{r-1}\left(\left(y_{i}\right)\right)\right) \leq 3$. 
(2) Assume that $U_{r}=c_{r 1} \perp c_{r 2}$ with $c_{r 1}, c_{r 2} \in \mathbb{Z}_{2}^{\times}$and $V_{r}=\varnothing$. Put

$$
B_{r-1}=U_{r-1}
$$

and

$$
B_{r-2}=\perp_{i=0}^{r-2} 2^{i} U_{i} \perp \perp_{i=1}^{r-1} 2^{i} V_{i}
$$

For $U_{r}$ put $\hat{U}_{r}=U_{r},-U_{r}, Y$, or $H$ according as $-c_{r 1} c_{r 2} \equiv-1 \bmod 8,-c_{r 1} c_{r 2} \equiv 3$ $\bmod 8,-c_{r 1} c_{r 2} \equiv 5 \bmod 8$, or $-c_{r 1} c_{r 2} \equiv 1 \bmod 8$. Then we have

$$
\begin{gathered}
\alpha_{2}(B, A)-2^{-m+n+1} \alpha_{2}\left(2^{r-1} \hat{U}_{r} \perp 2^{r-1} B_{r-1} \perp B_{r-2}, A\right) \\
=8^{-k_{r-1}-1} \sum_{\left(y_{i}\right)} f\left(2^{r-1} B_{r-1}\left(\left(y_{i}\right)\right) \perp B_{r-2}\right),
\end{gathered}
$$

where $\left(y_{i}\right)$ runs over all elements of $\left(\mathbb{Z}_{2} / 8 \mathbb{Z}_{2}\right)^{k_{r-1}+1}$ and $B_{r-1}\left(\left(y_{i}\right)\right)$ is a certain symmetric matrix of degree $k_{r-1}+1$ determined by $B_{r-1}$ and $\left(\left(y_{i}\right)\right)$ such that $\nu\left(\operatorname{det} B_{r-1}\left(\left(y_{i}\right)\right)\right)$ $\leq 3$.

(3) Assume that $V_{r}=Y$. Let

$$
B_{r-1}=2 U_{r}
$$

and

$$
B_{r-2}=\perp_{i=0}^{r-1} 2^{i}\left(U_{i} \perp V_{i}\right)
$$

Then we have

$$
\begin{gathered}
\alpha_{2}(B, A)-2^{-m+n+1} \alpha_{2}\left(2^{r-1}(1 \perp 3) \perp 2^{r-1} B_{r-1} \perp B_{r-2}, A\right) \\
=2^{-k_{r}-1} \sum_{\left(y_{i}\right)} f\left(B_{r-1}\left(\left(y_{i}\right)\right) \perp B_{r-2}\right),
\end{gathered}
$$

where $\left(y_{i}\right)$ runs over all elements of $\left(\mathbb{Z}_{2} / 8 \mathbb{Z}_{2}\right)^{k_{r}+1}$ and $B_{r-1}\left(\left(y_{i}\right)\right)$ is a certain symmetric matrix of degree $k_{r}+1$ determined by $B_{r-1}$ and $\left(\left(y_{i}\right)\right)$ such that $\nu\left(\operatorname{det} B_{r-1}\left(\left(y_{i}\right)\right)\right) \leq$ 3.

(4) Let $V_{r}=\overbrace{H \perp \cdots \perp H}^{l_{r}-1} \perp Y_{r}$ with $l_{r} \geq 1$. We set

$$
g=\alpha_{2}\left(2^{r} H \perp B_{r}, A\right)-2^{-m+n+1} \alpha_{2}\left(2^{r-1} H \perp B_{r}, A\right) .
$$

(4.1) Assume that $U_{r-1}=\varnothing$. Then

$$
\begin{gathered}
g=\left(1-2^{-m+n}\right)^{-1} \sum_{a}\left\{\left(2^{-3}-2^{-m+n-2}\right) f\left(2^{r+1} a \perp B_{r}\right)\right. \\
\left.+2^{-4} f\left(2^{r+2} a \perp B_{r}\right)+2^{-4} f\left(2^{r+3} a \perp B_{r}\right)\right\},
\end{gathered}
$$

where a runs over complete set of representatives of $\mathbb{Z}_{2}^{\times} /\left(\mathbb{Z}_{2}^{\times}\right)^{2}$. 
(4.2) Assume that $U_{r}=\left(c_{r}\right)$ or $c_{r 1} \perp c_{r 2}$. Then

$$
\begin{aligned}
g & =\sum_{a}\left\{2^{-3} f\left(2^{r} a \perp B_{r}\right)+\left(1-2^{-m+n}\right)^{-1}\left(2^{-4}-2^{-m+n+3}\right) f\left(2^{r+1} a \perp B_{r}\right)\right. \\
& \left.+2^{-5} f\left(2^{r+2} a \perp B_{r}\right)+2^{-5} f\left(2^{r+3} a \perp B_{r}\right)\right\}
\end{aligned}
$$

where a runs over complete set of representatives of $\mathbb{Z}_{2}^{\times} /\left(\mathbb{Z}_{2}^{\times}\right)^{2}$.

Proof. (1) First remark that $V_{r-1}=\perp_{i=1}^{l_{r-1}} H$ by (C.2) in the definition of canonical form. Put $B_{r-1}^{\prime}=2^{r-1}\left(U_{r-1} \perp V_{r-1}\right) \perp 2^{r-2} U_{r-2} \perp B_{r-2}$. By Theorem 3.2, we have

$$
\alpha_{2}\left(2^{r} c_{r} \perp B_{r-1}^{\prime}, A\right)=2^{-m+n+1} \alpha_{2}\left(2^{r-2} c_{r} \perp B_{r-1}^{\prime}, A\right)+f\left(2^{r} c_{r} \perp B_{r-1}^{\prime}\right) .
$$

Further we have

$$
a_{e}\left(n-1 ; 2^{r} c_{r} \perp B_{r-1}^{\prime}, A^{(1)}\right)=\sum_{\left(y_{i}\right) \in\left(\mathbb{Z}_{2} / 2^{e} \mathbb{Z}_{2}\right)^{n-1}} a_{e}\left(B_{r-1}^{\prime}+2^{r}\left(c_{r} y_{i} y_{j}\right)_{2 \leq i, j \leq n}, A^{(1)}\right),
$$

and $B_{r-1}^{\prime}+2^{r}\left(c_{r} y_{i} y_{j}\right)_{2 \leq i, j \leq n}$ is non-degenerate over $\mathbb{Z}_{p} / p^{e} \mathbb{Z}_{p}$ and its determinant does not depend on $e$ for a sufficiently large $e$ for any $\left(y_{2}, \ldots, y_{n}\right) \in \mathbb{Z}_{2}^{n-1}$. Thus we have

$$
\begin{gathered}
\alpha_{2}\left(n-1 ; 2^{r} c_{r} \perp B_{r-1}^{\prime}, A^{(1)}\right) \\
\sum_{\left(y_{i}\right) \in\left(\mathbb{Z}_{2} / 2^{e} \mathbb{Z}_{2}\right)^{n-1}} \alpha_{2}\left(B_{r-1}^{\prime}+2^{r}\left(c_{r} y_{i} y_{j}\right)_{2 \leq i, j \leq n}, A^{(1)}\right) .
\end{gathered}
$$

In particular, $B_{r-2}+2^{r}\left(c_{r} y_{i} y_{j}\right)_{l_{r-1}+k_{r-1}+k_{r-2}+2 \leq i, j \leq n}$ is equivalent, over $\mathbb{Z}_{2}$, to

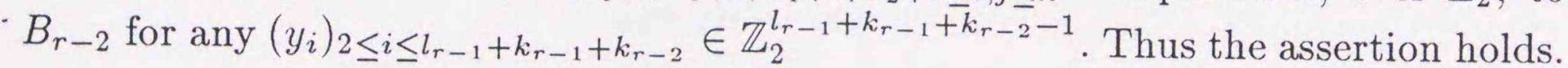

(2) For the matrix $U_{r}$, put $\tilde{U}_{r}=\left(\begin{array}{cc}c_{r 1}+c_{r 2} & c_{r 2} \\ c_{r 2} & 2 c_{r 2}\end{array}\right)$. Then $U_{r}$ is equivalent, over $\mathbb{Z}_{2}$ to $\tilde{U}_{r}$, and $2^{r} \tilde{U}_{r}\left[p^{-1} \perp 1\right]=2^{r-1} \hat{U}_{r}$. Thus, by applying Theorem 3.2 to $2^{r} U_{r} \perp B_{r-1} \perp B_{r-2}$ the assertion can be proved similalry to (1).

(3) The assertion can be proved similalry to (1) noting that $2^{r} Y\left[p^{-1} \perp 1\right]$ is equivalent to $2^{r-1}(1 \perp 3)$.

(4) Similarly to (3) of Theorem 4.1, we have

$$
\begin{gathered}
a_{e}\left(n-1 ; 2^{r} H \perp B_{r}, A^{(1)}\right)=\#\left\{\left(\begin{array}{c}
\left(y_{j}\right) \\
X
\end{array}\right) \in M_{m-1, n-1}\left(\mathbb{Z}_{2} / 2^{e} \mathbb{Z}_{2}\right) ;\right. \\
\left.A^{(1)}[X] \equiv\left(\begin{array}{cc}
2^{r+1} y_{1}-2^{2 r} B_{r}^{-1}\left[{ }^{t} \mathbf{y}^{\prime}\right] & 0 \\
0 & B_{r}
\end{array}\right) \bmod 2^{e}\right\},
\end{gathered}
$$

where ${ }^{t} \mathbf{y}^{\prime}=\left(y_{2}, \ldots, y_{n-1}\right)$.

(4.1) First let $\operatorname{deg}\left(U_{r}\right)=2$. By assumption, $2^{r+1} y_{1}-2^{2 r} B_{r}^{-1}\left[{ }^{t} \mathbf{y}^{\prime}\right] \in 2^{r+1} \mathbb{Z}_{2} / 2^{e} \mathbb{Z}_{2}$ for any $\left(y_{2}, \ldots, y_{n-1}\right) \in \mathbb{Z}_{2}^{n-1}$. Thus similarly to (3) of Theorem 3.1, we have

$$
a_{e}\left(n-1 ; 2^{r} H \perp B_{r}, A^{(1)}\right)=2^{e(n-2)+r+1} \sum_{a \in \mathbb{Z}_{2} / 2^{e-r-1} \mathbb{Z}_{2}} a_{e}\left(2^{r+1} a \perp B_{r}, A^{(1)}\right),
$$


thus the assertion holds.

(4.2) By assumption $2^{2 r} B_{r}^{-1}\left[{ }^{t} \mathbf{y}^{\prime}\right]=2^{r} \sum_{j=1}^{2} c_{r j} y_{2 l_{r}+j}^{2}+2^{r+1} b$ with $b \in \mathbb{Z}_{2}$ for any $\left(y_{1}, \ldots, y_{n}\right) \in \mathbb{Z}_{2}^{n}$. Thus $2^{r+1} y_{1}-2^{2 r} B_{r}^{-1}\left[{ }^{t} \mathbf{y}^{\prime}\right] \in 2^{r} \mathbb{Z}_{2}^{\times}$or $\in 2^{r+1} \mathbb{Z}_{2}$ according as exactly one of $y_{2 l_{r}+1}$ and $y_{2 l_{r}+2}$ is unit, or not. In the former case, for each such $\left(y_{2 l_{r}+1}, y_{2 l_{r}+2}\right) \in\left(\mathbb{Z}_{2} / 2^{e} \mathbb{Z}_{2}\right)^{2}$ and a fixed $c \in\left(2^{r} \mathbb{Z}_{2}^{\times}+2^{e} \mathbb{Z}_{2}\right) / 2^{e} \mathbb{Z}_{2}$ the mapping $\Phi_{c}$ from $\left(\mathbb{Z}_{2} / 2^{e} \mathbb{Z}_{2}\right)^{n-3}$ to $\{c\}$ defined by

$$
\Phi_{c}\left(\left(y_{1}, \ldots, y_{2 l_{r}}, y_{2 l_{r}+3}, \ldots, y_{n-1}\right)\right)=2^{r+1} y_{1}-2^{2 r} B_{r}^{-1}\left[{ }^{t} \mathbf{y}^{\prime}\right]
$$

is a surjection and $\Phi_{c}^{-1}(c)=2^{e(n-4)+r+1}$. In the latter case, for each such $\left(y_{2 l_{r}+1}, y_{2} l_{r}+2\right) \in$ $\left(\mathbb{Z}_{2} / 2^{e} \mathbb{Z}_{2}\right)^{2}$ the mapping $\Phi^{\prime}$ from $\left(\mathbb{Z}_{2} / 2^{e} \mathbb{Z}_{2}\right)^{n-3}$ to $2^{r+1} \mathbb{Z}_{2} / 2^{e} \mathbb{Z}_{2}$ defined by

$$
\Phi^{\prime}\left(\left(y_{1}, \ldots, y_{2 l_{r}}, y_{2 l_{r}+3}, \ldots, y_{n-1}\right)\right)=2^{r+1} y_{1}-2^{2 r} B_{r}^{-1}\left[{ }^{t} \mathbf{y}^{\prime}\right]
$$

is a surjection and for each $x \in 2^{r+1} \mathbb{Z}_{2} / 2^{e} \mathbb{Z}_{2}$ we have $\Phi^{\prime-1}(x)=2^{e(n-4)+r+1}$. Thus we have

$$
\begin{aligned}
& a_{e}\left(n-1 ; 2^{r} H \perp B_{r}, A^{(1)}\right) \\
& =2^{2 e-1+e(n-4)+r+1}\left\{\sum_{c \in\left(2^{r} \mathbb{Z}_{2}^{\times}+\mathbb{Z}_{2}\right) / 2^{e} \mathbb{Z}_{2}} a_{e}\left(c \perp B_{r}, A^{(1)}\right)\right. \\
& \left.+\sum_{a \in \mathbb{Z}_{2} / 2^{e-r-1} \mathbb{Z}_{2}} a_{e}\left(2^{r+1} a \perp B_{r}, A^{(1)}\right)\right\} \\
& =2^{e(n-1)-3} \sum_{a \in \mathbb{Z}_{2}^{\times} /\left(\mathbb{Z}_{2}^{\times}\right)^{2}} a_{e}\left(2^{r} a \perp B_{r}, A^{(1)}\right) \\
& +2^{e(n-2)+r} \sum_{a \in \mathbb{Z}_{2} / 2^{e-r-1} \mathbb{Z}_{2}} a_{e}\left(2^{r+1} a \perp B_{r}, A^{(1)}\right),
\end{aligned}
$$

thus the assertion follows similarly to (1.1) in this theorem. Similarly, the assertion holds for $\operatorname{deg}\left(U_{r}\right)=1$.

Corollary 4.4. Let $B_{1}, B_{2}$ and $B_{3}$ be non-degenerate symmetric matrices of level $2^{l_{1}}, 2^{l_{2}}$ and $2^{l_{3}}$, respectively, with entries in $\mathbb{Z}_{2}$. Put $B=2^{r} B_{1} \perp 2^{r-2} B_{2} \perp B_{3}$. Assume that $B$ is a canonical form, $B_{1}$ is a unimodular matrix of degree $\leq 2, l_{2} \leq 1$ and $r \geq l_{3}+1$. Put $\tilde{B}_{1}=2^{-1} B_{1}, \hat{B}_{1}, 1 \perp 3$, or $H$ according as $B_{1}=\left(b_{1}\right), \bar{b}_{1} \perp b_{2}, Y$, or $H$, where " $\hat{*}$ " is the same as in Theorem 4.3 (2). Then we have

$$
\begin{aligned}
\alpha_{2}(B, A) & -2^{-m+n+1} \alpha_{2}\left(2^{r-1} \tilde{B}_{1} \perp 2^{r-2} B_{2} \perp B_{3}, A\right) \\
= & \sum_{B_{2}^{\prime}} c\left(B ; B_{1}, B_{2}\right) f\left(2^{r-2} B^{\prime} \perp B_{3}\right),
\end{aligned}
$$

where $B^{\prime}$ runs over a certain finitely many symmetric matrices of degree $\operatorname{deg}\left(B_{1}\right)+$ $\operatorname{deg}\left(B_{2}\right)-1$, and $c\left(B^{\prime} ; B_{1}, B_{2}\right)$ is a rational number determined by $B_{1}, B_{2}$ and $A$ such that

$$
\sum_{B^{\prime}} c\left(B^{\prime} ; B_{1}, B_{2}\right)=1
$$




\section{Proof of the main theorems}

For non-degenerate symmetric matrices $A, B_{0}, B_{1}, \ldots, B_{s}$ of degree $m, n_{0}, n_{1}, \ldots, n_{s}$, respectively, with entries in $\mathbb{Z}_{p}$, define the formal power series $P\left(\left(B_{0}, B_{1}, \ldots, B_{s}\right), A ; x_{1}, \ldots, x_{s}\right)=\sum_{r_{1}, \ldots, r_{s}=0}^{\infty} \alpha_{p}\left(B_{0} \perp p^{r_{1}} B_{1} \perp \cdots \perp p^{r_{s}} B_{s}, A\right) x_{1}^{r_{1}} \ldots x_{s}^{r_{s}}$.

If $s=1$ and $\operatorname{deg}\left(B_{0}\right)=0$, the above series is nothing but the ones defined by Kitaoka in [9].

Let $\Delta_{p}$ be the complete set of representatives of $\mathbb{Z}_{p}^{\times} /\left(\mathbb{Z}_{p}^{\times}\right)^{2}$, and put

$$
\Lambda_{n, p}=\left\{\left(b_{0}, b_{1}, \ldots, b_{n}\right) ; b_{i} \in \Delta_{p}\right\}
$$

or

$$
\begin{gathered}
\left\{\left(B_{0}, B_{1}, \ldots, B_{s}\right) ; \operatorname{deg}\left(B_{0}\right)+\operatorname{deg}\left(B_{1}\right)+\cdots+\operatorname{deg}\left(B_{s}\right)=n,\right. \\
\left.B_{i}=\left(\begin{array}{ll}
0 & 1 \\
1 & 0
\end{array}\right),\left(\begin{array}{ll}
2 & 1 \\
1 & 2
\end{array}\right) \text { or } \operatorname{deg}\left(B_{i}\right)=1 \text { and } B_{i} \in \Delta_{p}\right\}
\end{gathered}
$$

according as $p \neq 2$ or $=2$. Then the set of power series

$$
\left\{P\left(\left(B_{0}, B_{1}, \ldots, B_{s}\right), A ; x_{1}, \ldots, x_{s}\right)\right\}_{\left(B_{0}, B_{1}, \ldots, B_{s}\right) \in \Lambda_{n, p}}
$$

gives complete information on the local densities $\alpha_{p}(B, A)$ for all $B$ of degree $n$ and $A$ of degree $m$. Therefore it is important to study these power series.

Definition. Let $B$ be a non-degenerate symmetric matrix with entries in $\mathbb{Z}_{p}$. $B$ is said to be maximal if there is no square matrix $X$ such that $\operatorname{det}(X) \in p \mathbb{Z}_{p}$ and $B\left[X^{-1}\right]$ is a symmetric matrix with entries in $\mathbb{Z}_{p}$.

To determine the denominator of formal power series, we need the followings.

Proposition 5.1(cf.[6]). Let $A$ be a symmetric unimodular matrix of degree $m$, $B_{1}$ and $B_{2}$ be symmetric matrices of degree $n_{1}, n_{2}$, respectively. Put $n=n_{1}+n_{2}$. Let $p^{b_{2}}$ be the level of $B_{2}$ if $n_{2}>0$. Put $l=\min \left(n_{1}-1, r\right)$, where $r$ is the Witt index of $A$, and $n=n_{1}+n_{2}$. Let $e$ be an integer such that $e \geq b_{2}+2 l+\delta_{2 p}+2$.

(1) Assume that $B_{1}$ is not maximal. Then there exists a maximal matrix $\tilde{B}_{1}$ such that $\operatorname{deg} B_{1}=\operatorname{deg} \tilde{B}_{1}$ and $\nu\left(\operatorname{det} B_{1}\right)-\nu\left(\operatorname{det} \tilde{B}_{1}\right)$ is a positive even integer, and

$$
\begin{aligned}
\alpha_{p}\left(p^{e} B_{1}\right. & \left.\perp B_{2}, A\right)-p^{(-m+n+1) b_{1}} \alpha_{p}\left(p^{e} \tilde{B}_{1} \perp B_{2}, A\right) \\
= & \sum_{B_{1}^{\prime}} c\left(B_{1}^{\prime}, B_{1}, A\right) f\left(p^{e-2} B_{1}^{\prime} \perp B_{2}\right),
\end{aligned}
$$

where $b_{1}=\left(\nu\left(\operatorname{det} B_{1}\right)-\nu\left(\operatorname{det} \tilde{B}_{1}\right)\right) / 2, B_{1}^{\prime}$ runs over finitely many symmetric matrices of degree $n_{1}-1$, and $c\left(B_{1}^{\prime}, B_{1}, A\right)$ is a rational number determined by $B_{1}^{\prime}, B_{1}$ and $A$ such that

$$
\sum_{B_{1}^{\prime}} c\left(B_{1}^{\prime}, B_{1}, A\right)=\frac{1-p^{(-m+n+1) b_{1}}}{1-p^{-m+n+1}} .
$$


(2) Assume that $B_{1}$ is maximal. Then we have

$$
\begin{aligned}
\alpha_{p}\left(p^{e} B_{1}\right. & \left.\perp B_{2}, A\right)-p^{(-m+n+1) n_{1}} \alpha_{p}\left(p^{e-2} B_{1} \perp B_{2}, A\right) \\
& =\sum_{B_{1}^{\prime}} c\left(B_{1}^{\prime}, B_{1}, A\right) f\left(p^{e-1} B_{1}^{\prime} \perp B_{2}\right),
\end{aligned}
$$

where $B_{1}^{\prime}$ runs over finitely many symmetric matrices of degree $n_{1}-1$, and $c\left(B_{1}^{\prime}, B_{1}, A\right)$ is a rational number determined by $B_{1}^{\prime}, B_{1}$ and $A$ such that

$$
\sum_{B_{1}^{\prime}} c\left(B_{1}^{\prime}, B_{1}, A\right)=\frac{1-p^{(-m+n+1) n_{1}}}{1-p^{-m+n+1}} .
$$
Proof. We note that a maximal matrix is equivalent, over $\mathbb{Z}_{p}$, to a matrix of the
following form:

$$
p U_{1} \perp U_{2} \perp V_{1},
$$

where $U_{1}, U_{2}$ and $V_{1}$ are the ones in section 1 , and in particular $\operatorname{deg}\left(U_{1}\right) \leq 1$ if $p=2$. Thus the assertion can be easily proved by using a Corollarry 4.2 or 4.4 ,
repeatedly. $\square$

For each non-negative integers $i, j$ and $k$ such that $1 \leq k \leq i$, put (cf. [4])

$$
\gamma(i, j, k)=(-1)^{k} \sum_{0 \leq l_{1}<\cdots<l_{k} \leq i-1} p^{\left(i-l_{1}\right)\left(j+l_{1}\right)} \ldots p^{\left(i-l_{k}\right)\left(j+l_{k}\right)} .
$$

Here we understand that $\gamma(i, j, 0)=1$. It is easy to see that

$$
\prod_{k=0}^{i-1}\left(1-p^{(i-k)(j+k)} x\right)=\sum_{k=0}^{i} \gamma(i, j, k) x^{k}
$$
Theorem 5.2. Let the notations and the assumptions be the same as in Proposition
5.1. Then we have

$$
\sum_{i=0}^{l+1} \gamma\left(n_{1},-m+n+1, i\right) \alpha_{p}\left(p^{e-2 i} B_{1} \perp B_{2}, A\right)=\phi \prod_{i=0}^{l} \frac{1-p^{\left(n_{1}-i\right)(-m+n+i+1)}}{1-p^{-m+n+i+1}} \psi(i),
$$

where

$$
\phi= \begin{cases}\beta_{p}\left(O_{l+1}, A\right) \alpha_{p}\left(B_{2}, A^{(l+1)}\right), & \text { (case I) } \\ p^{-2(l+1)} \alpha_{p}\left(B_{2}, A^{(l+1)}\right), & \text { (case II) } \\ p^{-(l+1)} \beta_{p}\left(O_{l+1}, A^{*}\right)\left\{\alpha_{p}\left(B_{2}, A^{(l+1)}\right)\right. & \\ \left.\quad+p^{-m-l+4}\left(p^{3(l+1)}-1\right)\left(p^{3}-1\right)^{-1} \beta_{p}\left(0, A^{*(l)}\right)^{-1} \alpha_{p}\left(B_{2}, A^{*(l)}\right)\right\}, & \text { (case III) }\end{cases}
$$

and

$$
\psi(i)= \begin{cases}1, & (\text { case I,III) } \\ \beta_{p}\left(0, A^{*(i)}\right)+\beta_{p}\left(t r(-U), A^{*(i)}\right), & (\text { case II })\end{cases}
$$


Here we make the convention that $\alpha_{p}\left(B_{2}, A^{(l+1)}\right)=1$ or 0 if $n=n_{1}$ or $l=r$,
pectively.

Proof. We shall prove (case III) only since the proof of (case I) is completed in [6] and (case II) can be proved in the same manner as in (case I).

We prove the assertion by induction on $n_{1}$. The assertion clearly holds if $n_{1}=1$. Assume that the assertion holds for $n_{1}-1$. First assume that $B_{1}$ is maximal. Then by (2) of Proposition 5.1, we have

$$
\begin{aligned}
& \alpha_{p}\left(p^{e} B_{1} \perp B_{2}, A\right)-p^{(-m+n+1) n_{1}} \alpha_{p}\left(p^{e-2} B_{1} \perp B_{2}, A\right) \\
& =p^{-1} \beta_{p}\left(0, A^{*}\right) \sum_{B_{1}^{\prime}} c\left(B_{1}^{\prime}, B_{1}, A\right) \alpha_{p}\left(p^{e-1} B_{1}^{\prime} \perp B_{2}, A^{(1)}\right) \\
& +p^{-m+3} \sum_{B_{1}^{\prime}} c\left(B_{1}^{\prime}, B_{1}, A\right) \alpha_{p}\left(p^{e-1} B_{1}^{\prime} \perp B_{2}, A^{*}\right),
\end{aligned}
$$
where $B_{1}^{\prime}$ and the others are ones in (2) of Proposition 5.1. By the inductive
hypothesis, we have

$$
\begin{gathered}
\sum_{i=0}^{l} \gamma\left(n_{1}-1,-m+n+2, i\right) \alpha_{p}\left(p^{e-2 i-1} B_{1}^{\prime} \perp B_{2}, A^{(1)}\right) \\
=p^{-l} \beta_{p}\left(O_{l}, A^{*(1)}\right)\left\{\alpha_{p}\left(B_{2}, A^{(l+1)}\right)+p^{-m-l+7}\left(p^{3 l}-1\right)\left(p^{3}-1\right)^{-1}\right. \\
\cdot \beta_{p}\left(0, A^{*(1)}\right)^{-1} \alpha_{p}\left(B_{2}, A^{*(l)}\right\} \prod_{i=0}^{l-1} \frac{1-p^{\left(n_{1}-i-1\right)(-m+n+i+2)}}{1-p^{-m+n+i+2}} .
\end{gathered}
$$

By applying (case II) and by noting that

$$
\beta_{p}(0, A) \beta_{p}\left(O_{i}, A^{(1)}\right)=\beta_{p}\left(O_{i+1}, A\right),
$$

we have

$$
\begin{gathered}
\sum_{i=0}^{l} \gamma\left(n_{1}-1,-m+n+2, i\right) \alpha_{p}\left(p^{e-2 i-1} B_{1}^{\prime} \perp B_{2}, A^{*}\right) \\
=p^{-2 l} \beta_{p}\left(O_{l}, A^{*}\right) \alpha_{p}\left(B_{2}, A^{*(l)}\right) \prod_{i=0}^{l-1} \frac{1-p^{\left(n_{1}-i-1\right)(-m+n+i+2)}}{1-p^{-m+n+i+2}}
\end{gathered}
$$

for any such $B_{1}^{\prime}$. Thus we have

$$
\sum_{i=0}^{l+1} \gamma\left(n_{1},-m+n+1, i\right) \alpha_{p}\left(p^{e-2 i} B_{1} \perp B_{2}, A\right)
$$

$=\sum_{i=0}^{l} \gamma\left(n_{1}-1,-m+n+2, i\right)\left\{\alpha_{p}\left(p^{e-2 i} B_{1}^{\prime} \perp B_{2}, A\right)-p^{-m+n+1} \alpha_{p}\left(p^{e-2 i-2} B_{1}^{\prime} \perp B_{2}, A\right)\right\}$ 


$$
\begin{aligned}
& =\sum_{i=0}^{l} \gamma\left(n_{1}-1,-m+n+2, i\right)\left\{p^{-1} \beta_{p}\left(0, A^{*}\right) \sum_{B_{1}^{\prime}} c\left(B_{1}^{\prime}, B_{1}, A\right) \alpha_{p}\left(p^{e-2 i-1} B_{1}^{\prime} \perp B_{2}, A^{(1)}\right)\right. \\
& \left.+p^{-m+3} \sum_{B_{1}^{\prime}} c\left(B_{1}^{\prime}, B_{1}, A\right) \alpha_{p}\left(p^{e-2 i-1} B_{1}^{\prime} \perp B_{2}, A^{*}\right)\right\} \\
& =p^{-1} \beta_{p}\left(0, A^{*}\right) \sum_{B_{1}^{\prime}} c\left(B_{1}^{\prime}, B_{1}, A\right) p^{-l} \beta_{p}\left(O_{l}, A^{*(1)}\right)\left\{\alpha_{p}\left(B_{2}, A^{(l+1)}\right)\right. \\
& +p^{-m-l+7}\left(p^{3 l}-1\right)\left(p^{3}-1\right)^{-1} \beta_{p}\left(0, A^{*(1)}\right)^{-1} \alpha_{p}\left(B_{2}, A^{*(l)}\right\} \prod_{i=0}^{l-1} \frac{1-p^{\left(n_{1}-i-1\right)(-m+n+i+2)}}{1-p^{-m+n+i+2}} \\
& +p^{-m+3} \sum_{B_{1}^{\prime}} c\left(B_{1}^{\prime}, B_{1}, A\right) p^{-2 l} \beta_{p}\left(O_{l}, A^{*}\right) \alpha_{p}\left(B_{2}, A^{*(l)}\right) \prod_{i=0}^{l-1} \frac{1-p^{\left(n_{1}-i-1\right)(-m+n+i+2)}}{1-p^{-m+n+i+2}} .
\end{aligned}
$$

Thus the assertion holds.

Next assume that $B_{1}$ is not maximal. Then by (1) of Proposition 5.1 there exists a maximal matrix $\tilde{B}_{1}$ such that we have

$$
\begin{gathered}
\alpha_{p}\left(p^{e} B_{1} \perp B_{2}, A\right)-p^{(-m+n+1) b_{1}} \alpha_{p}\left(p^{e-2} \tilde{B}_{1} \perp B_{2}, A\right) \\
=p^{-1} \beta_{p}\left(0, A^{*}\right) \sum_{B_{1}^{\prime}} c\left(B_{1}^{\prime}, B_{1}, A\right) \alpha_{p}\left(p^{e-2} B_{1}^{\prime} \perp B_{2}, A^{(1)}\right) \\
+p^{-m+3} \sum_{B_{1}^{\prime}} c\left(B_{1}^{\prime}, B_{1}, A\right) \alpha_{p}\left(p^{e-2} B_{1}^{\prime} \perp B_{2}, A^{*}\right)
\end{gathered}
$$
where $B_{1}^{\prime}$ runs over finitely many even matrices of degree $n_{1}-1$, and $c\left(B_{1}^{\prime}, B_{1}, A\right)$
is a rational number determined by $B_{1}^{\prime}$ and $A$ such that

$$
\sum_{B_{1}^{\prime}} c\left(B_{1}^{\prime}, B_{1}, A\right)=\frac{1-p^{(-m+n+1) b_{1}}}{1-p^{-m+n+1}}
$$

Thus we have

$$
\begin{aligned}
& \sum_{i=0}^{l+1} \gamma\left(n_{1},-m+n+1, i\right) \alpha_{p}\left(p^{e-2 i} B_{1} \perp B_{2}, A\right) \\
& =p^{(-m+n+1) b_{1}} \sum_{i=0}^{l+1} \gamma\left(n_{1},-m+n+1, i\right) \alpha_{p}\left(p^{e-2 i} \tilde{B}_{1} \perp B_{2}, A\right) \\
& +p^{-1} \beta_{p}\left(0, A^{*}\right) \sum_{B_{1}^{\prime}} c\left(B_{1}^{\prime}, B_{1}, A\right) \sum_{i=0}^{l+1} \gamma\left(n_{1},-m+n+1, i\right) \alpha_{p}\left(p^{e-2 i-2} B_{1}^{\prime} \perp B_{2}, A^{(1)}\right) \\
& +p^{-m+3} \sum_{B_{1}^{\prime}} c\left(B_{1}^{\prime}, B_{1}, A\right) \sum_{i=0}^{l+1} \gamma\left(n_{1},-m+n+1, i\right) \alpha_{p}\left(p^{e-2 i-2} B_{1}^{\prime} \perp B_{2}, A^{*}\right) .
\end{aligned}
$$


By the inductive hypothesis the second term on the right-hand side of the above is

$$
\begin{gathered}
\left(1-p^{(-m+n+1) b_{1}}\right) \prod_{i=0}^{l} \frac{1-p^{\left(n_{1}-i\right)(-m+n+i+1)}}{1-p^{-m+n+i+1}} p^{-(l+1)} \beta_{p}\left(O_{l+1}, A^{*}\right)\left\{\alpha_{p}\left(B_{2}, A^{(l+1)}\right)\right. \\
\left.+p^{-m-l+7}\left(p^{3 l}-1\right)\left(p^{3}-1\right)^{-1} \beta_{p}\left(0, A^{*(l)}\right)^{-1} \alpha_{p}\left(B_{2}, A^{*(l)}\right)\right\},
\end{gathered}
$$

and the third term on the right-hand side of the above is

$$
\left(1-p^{(-m+n+1) b_{1}}\right) \prod_{i=0}^{l} \frac{1-p^{\left(n_{1}-i\right)(-m+n+i+1)}}{1-p^{-m+n+i+1}} p^{-2 l_{m}+3} \beta_{p}\left(O_{l}, A^{*}\right) \alpha_{p}\left(B_{2}, A^{(l)}\right) .
$$

Thus the assertion holds.

Using Theorem 5.2, we obtain the following proposition.

Proposition 5.3. Let $A$ be as above. Let $B_{0}, B_{1}, \ldots, B_{s}$ be non-degenerate symmetric matices of degree $n_{0}, n_{1}, \ldots, n_{s}$, respectively, with entries in $\mathbb{Z}_{p}$. Let $p^{a_{i}}$ be the level of $B_{i}$ and $a_{1}<\cdots<a_{s}$. Put $l=\min \left(n_{1}, r\right)$ and $l_{0}=l+\delta_{2 p}$. Then we
have

$$
\begin{aligned}
& \prod_{i=0}^{l}\left(1-p^{\left(n_{1}-i\right)(-m+n+i+1)} x_{1}^{2}\right) Q\left(\left(B_{0}, B_{1}, \ldots, B_{s}\right), A ; x_{1}, \ldots, x_{s}\right) \\
& =\sum_{i=0}^{l_{0}} x_{1}^{2 i} \sum_{j=0}^{i} \gamma\left(n_{1},-m+n+1, i-j\right) \\
& \quad \cdot Q\left(\left(B_{0}, p^{2 j} B_{1} \perp B_{2}, B_{3}, \ldots, B_{s}\right), A ; x_{1} x_{2}, x_{3}, \ldots, x_{s}\right) \\
& +\sum_{i=0}^{l_{0}} x_{1}^{2 i+1} \sum_{j=0}^{i} \gamma\left(n_{1},-m+n+1, i-j\right) \\
& \quad \cdot Q\left(\left(B_{0}, p^{2 j+1} B_{1} \perp B_{2}, B_{3}, \ldots, B_{s}\right), A ; x_{1} x_{2}, x_{3}, \ldots, x_{s}\right) \\
& +\prod_{i=0}^{l} \frac{1-p^{\left(n_{1}-i\right)(-m+n+i+1)}}{1-p^{-m+n+i+1}} \frac{x_{1}^{2 l_{0}+2}}{1-x_{1}} \delta,
\end{aligned}
$$

where

$$
\delta= \begin{cases}\beta_{p}\left(O_{l+1}, A\right) Q\left(\left(B_{0}, B_{2}, \ldots, B_{s}\right), A^{(l+1)} ; x_{1} x_{2}, x_{3}, \ldots, x_{s}\right), & \text { (case I) } \\ p^{-2 l_{0}}\left\{\beta_{p}\left(0, A^{*(i)}\right)+\beta_{p}\left(\operatorname{tr}(-U), A^{*(i)}\right)\right\} & \text { (case II) } \\ & \quad Q\left(\left(B_{0}, B_{2}, \ldots, B_{s}\right), A^{\left(l_{0}\right)} ; x_{1} x_{2}, x_{3}, \ldots, x_{s}\right), \\ p^{-l_{0}} \beta_{p}\left(O_{l_{0}}, A^{*}\right)\left\{Q\left(\left(B_{0}, B_{2}, \ldots, B_{s}\right), A^{\left(l_{0}\right)} ; x_{1} x_{2}, x_{3}, \ldots, x_{s}\right)\right. & \\ & +p^{-m-l_{0}+4}\left(p^{3\left(l_{0}+1\right)}-1\right)\left(p^{3}-1\right)^{-1} \beta_{p}\left(0, A^{*\left(l_{0}\right)}\right)^{-1} \\ & \left.\cdot R\left(\left(B_{0}, B_{2}, \ldots, B_{s}\right), A^{*(l)} ; x_{1} x_{2}, x_{3}, \ldots, x_{s}\right)\right\},\end{cases}
$$

Here we make the convention that $Q\left(\left(B_{0}, p^{j} B_{1} \perp B_{2}, B_{3}, \ldots, B_{s}\right), A ; x_{1} x_{2}, x_{3}, \ldots, x_{s}\right)=$ $\alpha_{p}\left(B_{0} \perp p^{j} B_{1}, A\right)$ if $s=1$ and $Q\left(\left(B_{2}, \ldots, B_{s}\right), A^{(l+1)} ; x_{1} x_{2}, x_{3}, \ldots, x_{s}\right)=\alpha_{p}\left(B_{0}, A^{(l)}\right)$ or 0 if $s=1$ or $r=l$, respectively, and $j_{k}^{\prime}=j_{k}$ or $j_{k}+1$ according as $p \neq 2$ or not,
respectively. 
Theorem 5.4. Let $A$ be a symmetric unimodular matrix of degree $m$ and $B$ be a non-degenerate symmetric matrix of degree $n$. We assume that $B$ has the following decomposition: $B=B_{0} \perp B_{1} \perp \cdots \perp B_{s}, \operatorname{deg}\left(B_{i}\right)=n_{i}$. Let $t$ be the Witt index of A. Then $Q\left(\left(B_{0}, B_{1}, \ldots, B_{s}\right), A ; x_{1}, \ldots, x_{s}\right)$ is a rational function of $x_{1}, \ldots, x_{s}$ with
a denominator

$$
\prod_{k=1}^{s} \prod_{i=0}^{u_{k}}\left(1-p^{\left(n_{1}+\cdots+n_{k}-i\right)(-m+n+i+1)}\left(x_{1} \ldots x_{k}\right)^{2}\right) \prod_{k=1}^{s}\left(1-x_{1} \ldots x_{s}\right)^{v_{k}},
$$

where $u_{k}=\min \left(n_{1}+\cdots+n_{k}-1, t\right)$ and $v_{k}=1$ or 0 according as $t \geq n_{1}+\cdots+n_{k}$
or not.

Proof. It is directly proved by Proposition 5.3.

To prove the main Theorems, we need the following proposition due to Kat-
rada[5];

Proposition 5.5. Let $k, t$ and $l$ be non-negative integers such that $2 k \geq t \geq l \geq 1$ $k \geq l$ and $T=T_{1} \perp T_{2} . T_{1}$ and $T_{2}$ are non-degenerate even integral symmetric matrices of degree $l$ and $t-l$, respectively. Assume that $T_{1} \equiv O_{l} \bmod p$. Then we

$$
\begin{aligned}
& \beta_{p}\left(T_{1}, H_{k}\right) \alpha_{p}\left(T_{2}, H_{k-l} \perp\left(-T_{1}\right)\right) \\
& =\sum_{i=0}^{l}(-1)^{i} p^{i(i-1)+i(t+1-2 k)} \sum_{g_{i}} \alpha_{p}\left(T_{1}\left[g_{i}^{-1}\right] \perp T_{2}, H_{k}\right),
\end{aligned}
$$
where $H_{k}=H \perp \cdots \perp H(k$ copies $)$ and $g_{i}$ runs over the representatives of
$G L_{l}\left(\mathbb{Z}_{p}\right) \backslash G L_{l}\left(\mathbb{Z}_{p}\right) \lambda(i) G L_{l}\left(\mathbb{Z}_{p}\right)$ with $\lambda(i)=p E_{i} \perp E_{l-i}$.

Proof of Therem 1. Put $-p^{2} A=T_{1}$ and $p^{2} B=T_{2}$. Then by applying Proposition
5.4 with $k=l$, we have $\beta_{p}\left(-p^{2} A, H_{m}\right) \alpha_{p}\left(p^{2} B, p^{2} A\right)=\sum_{i=0}^{m}(-1)^{i} p^{i(i-1)+i(-m+n+1)} \sum_{g_{i}} \alpha_{p}\left(-p^{2} A\left[g_{i}^{-1}\right] \perp p^{2} B, H_{m}\right)$.

Noting that $\beta_{p}\left(-p^{2} A, H_{m}\right) \neq 0$ and $\alpha_{p}\left(p^{2} B, p^{2} A\right)=p^{n(n+1)} \alpha_{p}(B, A)$, we have

$$
\begin{aligned}
Q( & \left.\left(B_{0}, B_{1}, \ldots, B_{s}\right), A ; x_{1}, \ldots, x_{s}\right) \\
= & \beta_{p}\left(-p^{2} A, H_{m}\right)^{-1} \sum_{i=0}^{m}(-1)^{i} p^{i(-m+n+i)-n(n+1)} \\
& \cdot \sum_{g_{i}} Q\left(\left(-p^{2} A\left[g^{-1}\right] \perp p^{2} B_{0}, p^{2} B_{1}, \ldots, p^{2} B_{s}\right), H_{m} ; x_{1}, \ldots, x_{s}\right) .
\end{aligned}
$$

Then by applying Theorem 5.4 to $Q\left(\left(-p^{2} A\left[g^{-1}\right] \perp p^{2} B_{0}, p^{2} B_{1}, \ldots, p^{2} B_{s}\right), H_{m} ; x_{1}, \ldots, x_{s}\right)$ 's,
we complete the proof. 
To prove Theorem 2, define

$$
\begin{gathered}
\tau(s)=\left\{\left(r_{i_{1}}, \ldots, r_{i_{s}}\right) \in\left(\mathbb{Z}_{\geq 0}\right)^{s} ; r_{i_{a}} \geq r_{i_{b}} \text { or } r_{i_{a}}>r_{i_{b}}\right. \\
\text { according as } \left.i_{a}<i_{b} \text { or not for } 1 \leq a, b \leq s .\right\} .
\end{gathered}
$$

Let $A$ be the non-degenerate matrix of degree $m$ with the Witt index $t, B_{0}, B_{1} \ldots, B_{s}$ be as above. Define

$$
\begin{aligned}
& Q_{i_{1}, \ldots, i_{s}}\left(\left(B_{0}, B_{1}, \ldots, B_{s}\right), A ; x_{1}, \ldots, x_{s}\right) \\
& \quad=\sum_{\left(r_{i_{1}}, \ldots r_{i_{s}}\right) \in \tau(s)} \alpha_{p}\left(B_{0} \perp p^{r_{i_{1}}} B_{1} \perp \cdots \perp p^{r_{i_{s}}} B_{s}, A\right) x_{1}^{r_{i_{1}}} \ldots x_{s}^{r_{i_{s}}} .
\end{aligned}
$$

Theorem 5.6. Let the notation be as above. Then $Q_{i_{1}, \ldots, i_{s}}\left(\left(B_{0}, B_{1}, \ldots, B_{s}\right)\right.$, $\left.A ; x_{1}, \ldots, x_{s}\right)$ is a rational function of $x_{1}, \ldots, x_{s}$ with a denominator

$$
\prod_{k=1}^{s} \prod_{i=0}^{u_{k}}\left(1-p^{\left(n_{1}+\cdots+n_{k}-i\right)(-m+n+i+1)}\left(x_{1} \ldots x_{k}\right)^{2}\right) \prod_{k=1}^{s}\left(1-x_{1} \ldots x_{s}\right)^{v_{k}}
$$
where $u_{k}=\min \left(n_{1}+\cdots+n_{k}-1, t\right)$ and $v_{k}=1$ or 0 according as $t \geq n_{1}+\cdots+n_{k}$
or not.

Proof. This can be easily proved by induction on $s$ as that in Theorem 1 by noting that Proposition 5.3 is effective for $Q_{i_{1}, \ldots, i_{s}}\left(\left(B_{0}, B_{1}, \ldots, B_{s}\right), A ; x_{1}, \ldots, x_{s}\right)$ instead of $Q\left(\left(B_{0}, B_{1}, \ldots, B_{s}\right), A ; x_{1}, \ldots, x_{s}\right)$.

Now, we can prove Theorem 2.

proof of Theorem 2. Identifying $\left(i_{1}, \ldots, i_{s}\right)$ and $\left(\begin{array}{ccc}1 & \ldots & s \\ i_{1} & \ldots & i_{s}\end{array}\right) \in \mathfrak{S}_{s}($ symmetric group of degree $s$ ), we can write

$P\left(\left(B_{0}, B_{1}, \ldots, B_{s}\right), A ; x_{1}, \ldots, x_{s}\right)=\sum_{\left(i_{1}, \ldots, i_{s}\right) \in \mathfrak{S}_{s}} Q_{i_{1}, \ldots, i_{s}}\left(\left(B_{0}, B_{1}, \ldots, B_{s}\right), A ; x_{1}, \ldots, x_{s}\right)$,

that is, $P\left(\left(B_{0}, B_{1}, \ldots, B_{s}\right), A ; x_{1}, \ldots, x_{s}\right)$ is a finite linear combination of $Q_{i_{1}, \ldots, i_{s}}\left(\left(B_{0}, B_{1}, \ldots, B_{s}\right), A ; x_{1}, \ldots, x_{s}\right)$, which proves the assertion.

\section{REFERENCES}

[1] Böcherer, S. and Sato, F., Rationality of certain formal power series related to local densities, Comment. Math. Univ. St. Paul. 36 (1987), pp 53-86

[2] Hironaka, Y., On a denominator of Kitaoka's formal power series attached to local densities, Comment. Math. Univ. St. Paul. 37 (1988), pp 159-171

[3] Katsurada, H., A certain formal power series of several variables attached to 
local densities of quadratic forms I, J. of Number Theory 51 (1995), pp 169-209

[4] Katsurada, H., A certain formal power series of several variables attached to local densities of quadratic forms II, Proc. Japan Acad. 70 Ser.A (1994), pp 208-211

[5] Katsurada, H., An explicit formula for the Fourier coefficient of Siegel-Eisenstein series, preprint

[6] Katsurada, H. and Hisasue, M., A recursion formula for local densities, to appear in J. of Number Theory

[7] Katsurada, H. and Hisasue, M., A remark on a certain power series attached to local densities, preprint

[8] Kitaoka, Y., Dirichlet series in the theory of quadratic forms, Nagoya Math. J. 95 (1984), pp 73-84

[9] Kitaoka, Y., Local densities of quadratic forms and Fourier coefficients of Eisenstein series, Nagoya Math. J. 103 (1986), pp 149-160

[10] Kitaoka, Y., Arithmetic of quadratic forms, Cambridge University press (1993)

[11] Siegel, C. L., Über die analytische Theorie der quadratischen Formen, Ann. of Math. 36 (1935), pp 527-606

[12] Watson, G. L., The 2-adic density of a quadratic form, Mathematica 23 (1976), pp 94-106 


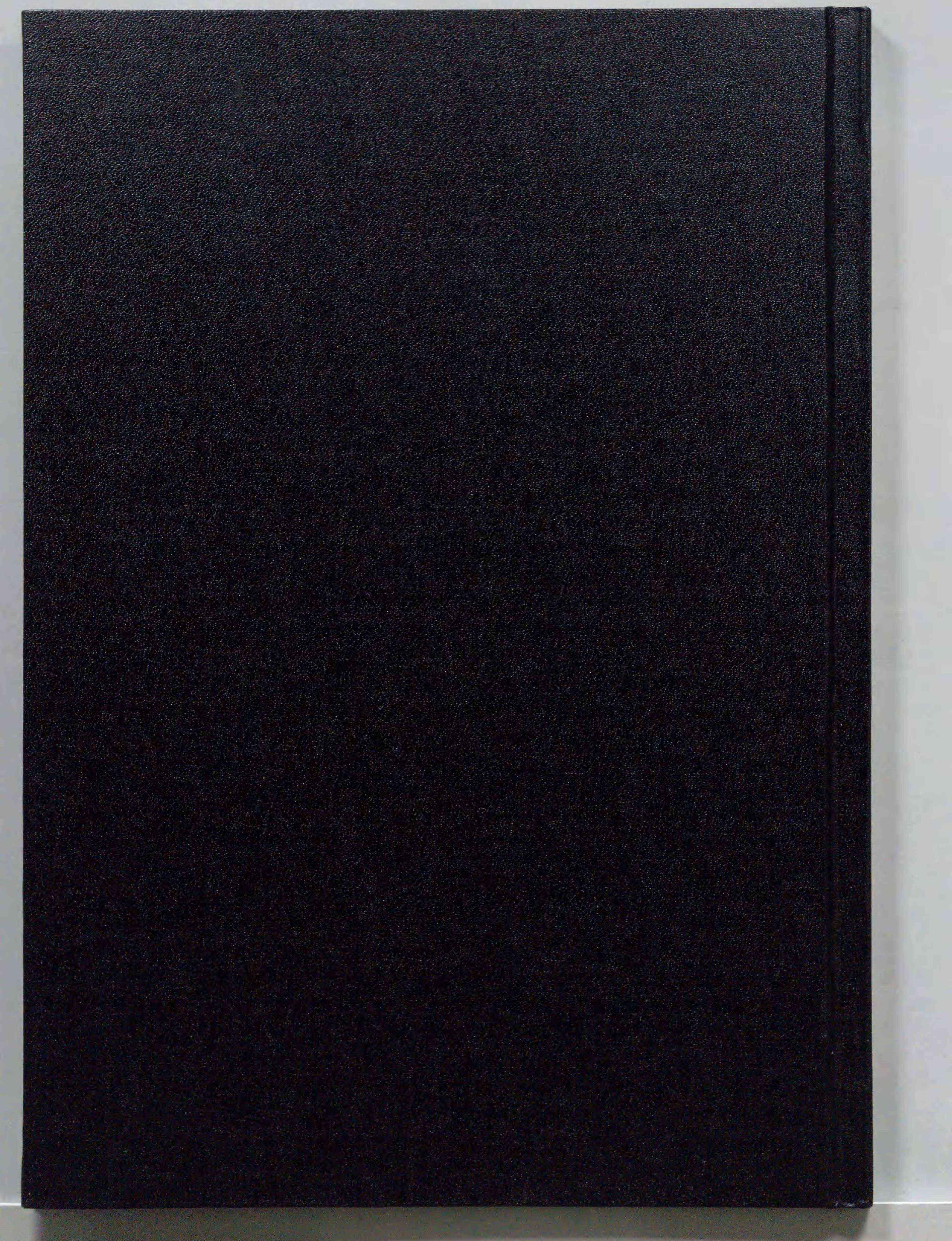




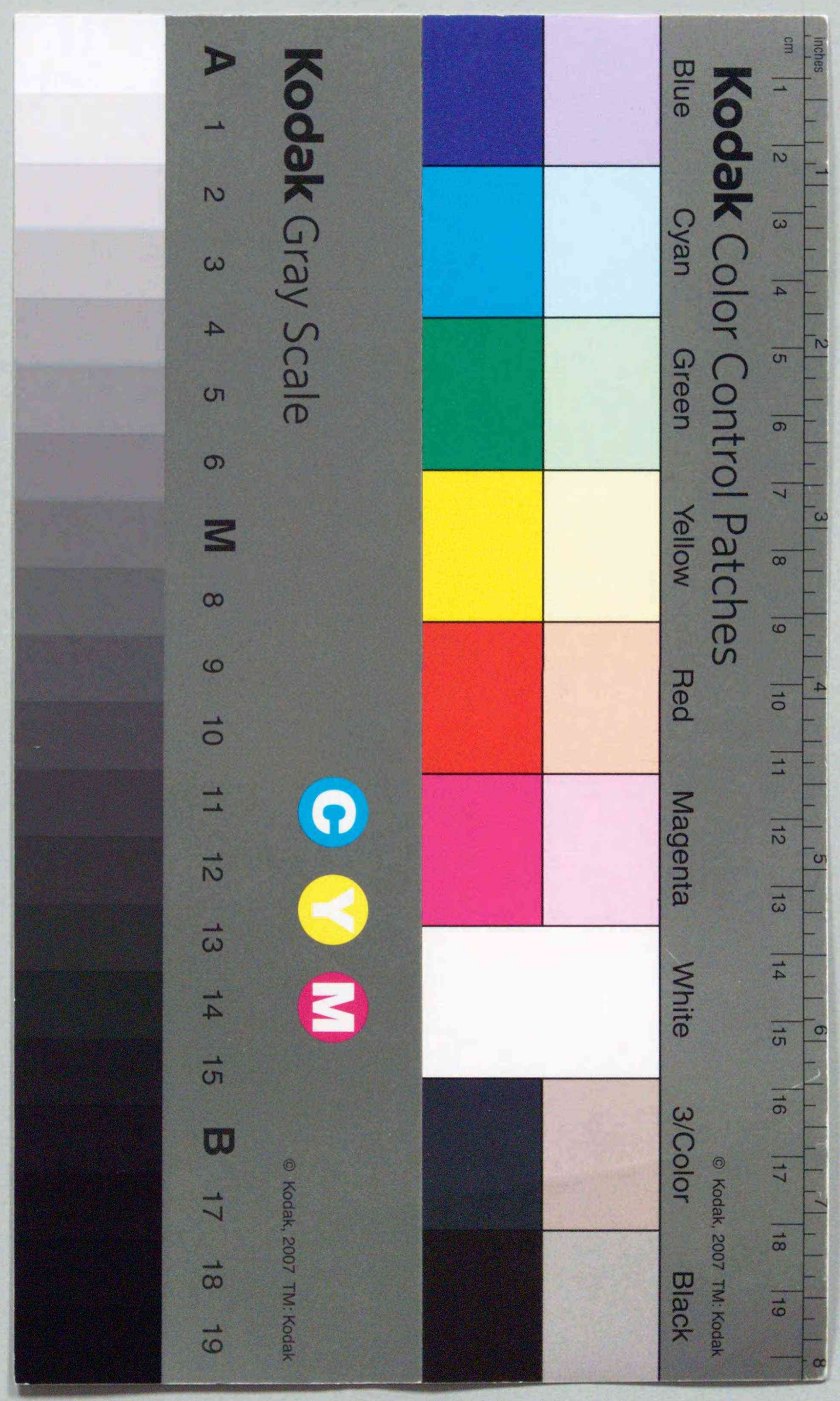

